\title{
1 STRUCTURAL EFFECTS OF MICROALGAE ADDITIVES ON THE STARCH \\ 2 GELATINISATION PROCESS
}

3

4 Marta Martínez-Sanz*, María José Fabra, Laura G. Gómez-Mascaraque and Amparo López-Rubio 5

6

7 Food Safety and Preservation Department, IATA-CSIC, Avda. Agustin Escardino 7, 46980

8 Paterna, Valencia (Spain).

9

10 *Corresponding author: Tel.: +34 963200022; fax: +34 963636301

11 E-mail address: mmartinez@iata.csic.es

12 


\section{Abstract}

14 This work presents a detailed structural characterisation of the starch gelatinisation process 15 and the effect of the addition of three microalgae species, Nannochloropsis gaditana sp., 16 Scenedesmus almeriensis and Spirulina, by means of an advanced approach consisting of 17 temperature-resolved simultaneous SAXS/WAXS experiments, combined with DSC. 18 Furthermore, regular and high amylose corn starch were utilised to evaluate the impact of 19 the amylose content.

20 The presence of microalgae has been seen to limit water accessibility towards the interior of 21 starch granules, reducing granule swelling and, thus, hindering the arrangement of 22 amylopectin helices into highly ordered crystalline structures. As a result, more 23 heterogeneous lamellar structures, with reduced apparent crystallinity, are attained. Despite 24 the existence of lipidic compounds in the three microalgae species, the tough cell walls in 25 Nannochloropsis and Scenedesmus impede their release towards the aqueous medium. In 26 contrast, the weak cell walls in Spirulina are disrupted by stirring, allowing cell components 27 to be released. The diffused lipids form helical inclusion complexes with the amylose chains 28 and promote the crystallisation of V-type structures. The presence of amylose-lipid 29 complexes counteracts the limited water swelling effect and results in the formation of more 30 crystalline and homogeneous lamellar structures. This result is relevant for the food industry 31 due to the potential of Spirulina to affect the processability and nutritional characteristics of 32 starch-based products.

33

34 Keywords: starch; microalgae; gelatinisation; SAXS; WAXS; DSC 35 


\section{1. Introduction}

37 Starch is one of the most widely known dietary polysaccharides since it is the main 38 component in raw and processed foods. Native starch is found in vegetal resources forming 39 water-insoluble semi-crystalline granules which present a complex hierarchical architecture 40 characterised by at least four structural levels: (i) granules, (ii) semi-crystalline amorphous 41 growth rings, (iii) lamellae (i.e. stacks of alternating amorphous and crystalline structures) 42 and (iv) linear amylose and branched amylopectin chains. The crystalline regions are thought 43 to consist mainly of amylopectin side chains which are organized in double helices (Imberty, 44 Buléon, Tran, \& Pérez, 1991), whereas the amorphous regions are mainly composed of linear 45 amylose chains and less ordered amylopectin branch points (Pérez \& Bertoft, 2010). 46 Depending on several factors such as the amylose/amylopectin ratio and the amylopectin 47 chain length, these helices can be arranged differently into A-type (mainly found in cereal 48 starches and related to short double helices) or B-type (long double helices found in tubers 49 and high amylose starches) crystalline unit cells (Cheetham \& Tao, 1998; Hoover, 2001; 50 Paul J Jenkins, Cameron, \& Donald, 1993; Salman, et al., 2009). Water has a great influence 51 in the long range order of starches, constituting up to $4-7 \%$ and $25-27 \%$ of the crystalline 52 unit cell in the A and B polymorphs, respectively (Imberty, Chanzy, Pérez, Bulèon, \& Tran, 53 1988; Imberty \& Perez, 1988; Sarko \& Wu, 1978).

54

55 Many food processing methods involve the use of combined heat and humidity, leading to 56 starch gelatinisation which involves the break-up of the starch structure. Essentially, the 57 gelatinisation process is initiated by the access of water towards the interior of starch 58 granules, which swell and absorb water. Subsequently, structural changes occur at the 59 different architectural levels, leading to crystallinity losses, disruption of the crystalline and 60 lamellar structure of starch and amylose molecules leaking out of the starch granules forming 
61 a continuous gel. This complex gelatinisation phenomenon is governed by a number of 62 factors such as starch botanical origin, plant growth conditions, extraction methods, water 63 content, presence of additives, heating rate and thermal history (Imberty, et al., 1991; Liu, 64 Yu, Xie, \& Chen, 2006; Tester \& Morrison, 1990; Varavinit, Shobsngob, Varanyanond, 65 Chinachoti, \& Naivikul, 2003; Waigh, Gidley, Komanshek, \& Donald, 2000). A detailed 66 understanding of the structural changes taking place during starch gelatinisation, at the 67 different length-scales, as well as the effect of different parameters, is highly relevant to 68 several scientific and industrial fields. For instance, in many food processing methods such 69 as baking of bread, extrusion of cereal-based products, thickening, and gelling of sauces, 70 starch gelatinisation is a key factor to produce a desirable texture or consistency of the end 71 product (Biliaderis, Maurice, \& Vose, 1980). From the human nutrition perspective, the 72 degree of starch gelatinisation in food products is known to affect its digestion rate (Joergen 73 Holm, Lundquist, Björck, Eliasson, \& Asp, 1988). Furthermore, the gelatinisation process is 74 the basis for the processing methods applied to produce starch-based biopolymeric materials 75 (Li, et al., 2011; Liu, Xie, Yu, Chen, \& Li, 2009).

76

77 Microalgae constitute one the most promising sustainable feedstocks for the manufacture 78 of plant-derived products. Due to their ease of cultivation, high growing rates and 79 productivity and possibility of adapting the harvesting conditions to modify their 80 composition, microalgae have gained a great deal of interest for their use in a wide range 81 of applications such as the production of biofuels (Mata, Martins, \& Caetano, 2010) and 82 food commodities (Draaisma, et al., 2013; Spolaore, Joannis-Cassan, Duran, \& Isambert, 83 2006). Their interest from the human nutrition perspective lays in their interesting chemical 84 composition. In particular, the high protein content of several species and their interesting 85 lipidic profile, with relatively high amounts of $\omega 3$ and $\omega 6$ fatty acids (Spolaore, et al., 2006), 
86 make them attractive for the production of nutritional supplements or food additives. The 87 incorporation of microalgae into pasta and bakery products to enhance their nutritional 88 profile is currently being investigated and has been reported in several recent works (De 89 Marco, Steffolani, Martínez, \& León, 2014; Monica Fradique, et al., 2010; Mónica 90 Fradique, et al., 2013; Kadam \& Prabhasankar, 2010). The components present in the 91 microalgae may interfere with the gelatinisation process in starchy foods and, thus, the 92 processing conditions may also be affected. Therefore, investigating the effect of different 93 microalgae on the gelatinisation of starch may be of interest to determine their impact on 94 the processability and digestibility of starch-microalgae blends. In this work, we have 95 investigated the structural changes undergone by two starches, with different amylose 96 contents, as well as the effect of three different microalgae species, during the gelatinisation 97 process. To this end, an advanced approach of temperature-resolved simultaneous small 98 angle and wide angle X-ray scattering (SAXS/WAXS) experiments, combined with DSC 99 characterisation, has been utilised. The use of scattering techniques is particularly 100 advantageous to study the starch gelatinisation process, since it enables the characterisation 101 of different structural levels (crystalline and lamellar structures) in starch as the temperature 102 is raised and at high relative humidity conditions.

103

\section{2. Materials and methods}

\section{2.1 Materials}

106 Corn starch (27-28\% amylose) and high amylose starch (70\% amylose) powders were 107 supplied by Roquette (Roquette Laisa España, Benifaió, Spain). The three different 108 microalgae species, i.e. Nannocloropsis gaditana sp., Spirulina and Scenedesmus 109 almeriensis, in the form of dry powders, were kindly donated by Dr. Acién from the 110 University of Almeria (Spain). 


\section{2.2 TEM characterisation of microalgae cell walls}

113 The raw microalgae were dispersed in water by vortex stirring at a concentration of $4 \mathrm{~g} / \mathrm{L}$. 114 One drop ( $8 \mu \mathrm{l})$ of the prepared suspensions was allowed to dry on a carbon-coated grid (200 115 mesh). The microalgae cell walls were stained with a $2 \%(w / w)$ solution of uranyl acetate. 116 TEM was performed using a JEOL 1010 equipped with a digital Bioscan (Gatan) image 117 acquisition system at $80 \mathrm{kV}$.

118

\section{2.3 Preparation of starch and starch/microalgae dispersions}

120 Starch dispersions (samples designated as “corn” and "amylo” for the corn starch and high 121 amylose starch, respectively) were prepared by adding $0.5 \mathrm{~g}$ of starch into $1 \mathrm{~mL}$ of water 122 and subjecting the samples to vortex stirring for $2 \mathrm{~min}$. For the starch/microalgae samples, $1230.004 \mathrm{~g}$ of the microalgae powder were also added prior to stirring. The dispersions were 124 immediately used for the SAXS/WAXS and the DSC experiments.

125

\section{2.4 Temperature resolved SAXS/WAXS experiments}

127 Combined small and wide angle X-ray scattering (SAXS and WAXS, respectively) 128 experiments were carried out in the Non Crystalline Diffraction beamline, BL-11, at ALBA 129 synchrotron light source (www.albasynchrotron.es). The starch and starch/microalgae 130 dispersions were placed in sealed 2 mm quartz capillaries (Hilgenburg Gmbh, Germany).

131 The energy of the incident photons was $12.4 \mathrm{KeV}$ or equivalently a wavelength, $\lambda$, of $1 \AA$. 132 The SAXS diffraction patterns were collected by means of a 9 CCD detector Quantum 133 ADSC 315r with an active area of 315 x 315 mm$^{2}$, an effective pixel size of 102 x $102 \mu m^{2}$ 134 and a dynamic range of 16 bits. The sample-to-detector distance was set to $6488 \mathrm{~mm}$, 135 resulting in a q range with a maximum value of $q=0.25 \AA^{-1}$. Additionally, the WAXS 
136 diffraction patterns were collected by means of a 3 CCD detector Rayonix LX255-HS with 137 an active area of $85 \times 255 \mathrm{~mm}^{2}$, an effective pixel size of $44 \times 44 \mu^{2}$ and a dynamic range 138 of 16 bits. In this case, the sample-to-detector distance was set to $144.9 \mathrm{~mm}$, corresponding 139 to a maximum q value of $7.87 \AA^{-1}$. This detector was tilted with a pitch of 26.4 degrees. 140 Based on previous experiments, an exposure time, of 2 seconds was selected for both 141 detectors.

142

143 Samples were heated from $30{ }^{\circ} \mathrm{C}$ to $110^{\circ} \mathrm{C}$ at a heating rate of $2{ }^{\circ} \mathrm{C} / \mathrm{min}$. Data were collected 144 in frames of 30 seconds, followed by a period of 30 seconds in which the samples were 145 protected from the beam by a local shutter. Each data frame thus corresponds to a 146 temperature range of $1{ }^{\circ} \mathrm{C}$, with one data frame every $2{ }^{\circ} \mathrm{C}$. The data reduction was treated 147 by pyFAI python code (ESRF) (Kieffer \& Wright, 2013), modified by ALBA beamline 148 staff, to do on-line azimuthal integrations from a previously calibrated file. The calibration 149 files were created from well-known standards, i.e. Silver behenate (AgBh) and $\mathrm{Cr}_{2} \mathrm{O}_{3}$ for 150 SAXS and WAXS respectively. The intensity profiles were then represented as a function 151 of $\mathrm{q}$ (SAXS) and $2 \theta$ (WAXS) using the IRENA macro suite (Ilavsky \& Jemian, 2009) 152 within the Igor software package (Wavemetrics, Lake Oswego, Oregon).

153

\subsection{SAXS/WAXS data fitting}

155 SAXS data were fitted using the Igor NIST analysis macro suite (Kline, 2006) and applying 156 a mathematical function consisting of a power-law term plus one Gaussian-Lorentzian 157 peak, similar to that previously reported for several starch samples (A. Lopez-Rubio, et al., 158 2007; Salman, et al., 2009): 
$159 I(q)=A \cdot q^{-m}+\left[R \cdot\left[I_{\max } \cdot\left(1+\left(\frac{2 \cdot\left(q-q_{\max }\right)}{\Delta q}\right)^{2}\right)^{-1}\right]\right]+\left[(1-R) \cdot\left[I_{\max } \cdot \exp \left[-\frac{1}{2}\right.\right.\right.$.

$\left.\left.\left.160\left(\frac{q-q_{\max }}{\Delta q}\right)^{2}\right]\right]\right]+b k g$

161 The first term in equation (1) corresponds to the power-law function (where $A$ is a prefactor 162 and $m$ is the power-law exponent) to account for the underlying diffuse scattering, the 163 second and third terms correspond to the Lorentzian and Gaussian functions used to 164 describe the starch lamellar peak (where $q_{\max }$ is the peak position, $I_{\max }$ is the intensity of 165 the peak, $\Delta q$ is the full width at half maximum and $R$ is the Lorentzian to Gaussian ratio of 166 the peak shape) and the fourth term accounts for the incoherent background.

167

168 WAXS peak fitting was performed in Igor, following the procedure described in a previous 169 work (Amparo Lopez-Rubio, Flanagan, Gilbert, \& Gidley, 2008). The obtained values 170 from the fitting coefficients are those that minimize the value of Chi-squared, which is 171 defined as:

$172 \chi^{2}=\sum\left(\frac{y-y_{i}}{\sigma_{i}}\right)^{2}$

173 where $\mathrm{y}$ is a fitted value for a given point, $y_{i}$ is the measured data value for the point and $174 \sigma_{i}$ is an estimate of the standard deviation for $y_{i}$. The curve fitting operation is carried out 175 iteratively and for each iteration, the fitting coefficients are refined to minimize $\chi^{2}$. The 176 crystallinity index $\mathrm{X}_{\mathrm{C}}$ was determined from the obtained fitting results by applying the 177 following equation:

$178 X_{C}(\%)=\frac{\sum A_{\text {Crystal }}}{A_{\text {Total }}} \times 100$

179 where $A_{\text {Total }}$ is the sum of the areas under all the diffraction peaks and $\Sigma A_{\text {Crystal }}$ is the sum of 180 the areas corresponding to the crystalline peaks. 


\section{2.6 Differential Scanning Calorimetry (DSC)}

183 DSC measurements of starch and starch/microalgae dispersions were performed on a Perkin184 Elmer DSC 8000 thermal analysis system using $\mathrm{N}_{2}$ as the purging gas. Approximately 10 $185 \mathrm{mg}$ of samples were weighted and added into hermetically sealed aluminium sample pans. 186 The sample treatment consisted of heating step from $0{ }^{\circ} \mathrm{C}$ to $180{ }^{\circ} \mathrm{C}$ at a heating rate of 2 $187^{\circ} \mathrm{C} / \mathrm{min}$. Before evaluation, similar runs of an empty pan were subtracted from the 188 thermograms. The DSC equipment was calibrated using indium as a standard. Measurements 189 were done, at least, in triplicate.

190

\section{3. Results and Discussion}

\section{3.1 Thermodynamic characterisation of the starch gelatinisation process}

193 The gelatinisation process of starch and starch-microalgae suspensions in excess water was 194 studied by means of DSC characterisation by heating the samples at a rate of $2^{\circ} \mathrm{C} / \mathrm{min}$. 195 Typical DSC profiles for the corn starch and high amylose based samples are shown in 196 Figures 1A and 1B, respectively, and the extracted gelatinisation parameters are summarised 197 in Table 1. All the samples show a broad melting peak that corresponds to the gelatinisation 198 endotherm. In the case of corn starch the endotherm extends over a temperature range of ca. $19960-70^{\circ} \mathrm{C}$ with the maximum at $64^{\circ} \mathrm{C}$, whereas the process takes place within the range of ca. $20070-90^{\circ} \mathrm{C}$, with the maximum at $78^{\circ} \mathrm{C}$, for the high amylose starch. Similar gelatinisation 201 temperatures have been previously reported for corn starch (Liu, et al., 2006; Yoshimura, 202 Takaya, \& Nishinari, 1996) and high amylose starch (Liu, et al., 2006) samples. The higher 203 gelatinisation temperature for the high amylose starch is partly a result of the amylose 204 fraction being less prone to swelling than the amylopectin (Hermansson \& Svegmark, 1996; 205 Yuryev, Kalistratova, van Soest, \& Niemann, 1998). Moreover, despite the higher packing 
206 density of the A-type polymorph, B-type starches have been seen to be more enzymatically 207 resistant than A-type starches due to the greater crystallinity of the former ones in the outer 208 granule regions (Sevenou, Hill, Farhat, \& Mitchell, 2002). This could be an additional factor 209 limiting water accessibility and therefore, hindering the gelatinisation process in high 210 amylose starch.

211

212 It should be noted that the gelatinisation endotherm clearly presents a distinct shape of two 213 overlapped peaks in the case of corn starch (see inset in Figure 1A). A similar behaviour has 214 been previously reported for corn starch samples (46\% w/w suspensions in water) with 215 comparable amylose content (Biliaderis, Maurice \& Vose, 1980). This is related to the 216 existence of two different transitions that have been described to take place in the presence 217 of moderate/high water contents: (i) the disruption of the inter-molecular hydrogen bonds 218 leading to the dissociation of the amylopectin double helices from the sides of the 219 crystallites, known as the smectic to nematic/isotropic phase transition and (ii) the unwinding 220 of the amylopectin helices at higher temperatures, known as the helix-coil transition (Waigh, 221 Gidley, Komanshek \& Donald, 2000). In the case of the high amylose starch, the 222 gelatinisation transition is characterised by a broad peak, similar to that previously detected 223 for corn starch with 70\% amylose content. This broad peak has been described to account 224 for several processes: the disruption of amylopectin double helices, the dissociation of the 225 amylose-lipid complexes and the melting of single-helical V-type crystallites (Matveev, et 226 al., 2001).

228 Both types of starch present a similar gelatinisation enthalpy, although the peak height index 229 (PHI), defined as the ratio between the enthalpy and the difference between the peak 230 maximum and onset temperatures (Krueger, Knutson, Inglett, \& Walker, 1987), which 
231 provides a measure of the uniformity in the gelatinisation process, is greater for corn starch. 232 This suggests that although a comparable amount of energy is needed to melt the 233 amylopectin crystallites in both samples, a more heterogenous process takes place in the case 234 of the high amylose starch, supporting the co-existence of different types of crystallite 235 structures.

236

237 As deduced from Figure 1A and Table 1, the incorporation of microalgae into the corn starch 238 suspensions does not lead to significant changes on the gelatinisation parameters estimated 239 from the DSC thermograms. In contrast, the presence of microalgae seems to induce an 240 increase in the maximum of the gelatinisation endotherm for the high amylose starch 241 samples, although given the large associated standard deviation values the differences cannot 242 be considered as significative. This effect is more evident for the Spirulina and Scenedesmus 243 species and in the latter, it is accompanied by an increase in the gelatinisation enthalpy and 244 the peak height index (i.e. a larger amount of energy is required to complete the phase 245 transition and the gelatinisation process is more homogeneous). These results suggest that 246 certain compounds in the microalgae are able to interact with the amylose fraction in the 247 high amylose starch promoting the formation of more thermodynamically stable 248 suspensions. 


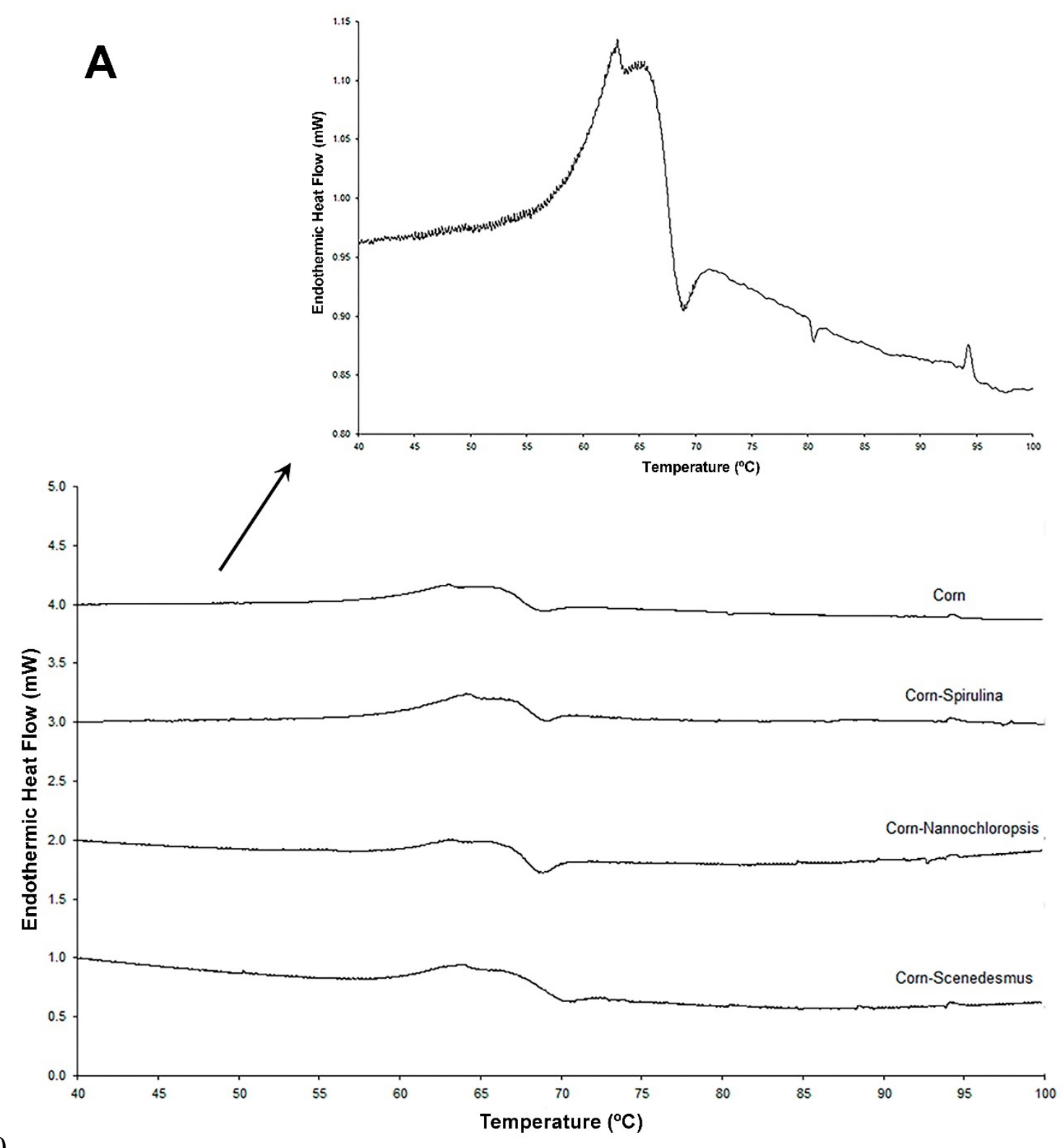


B

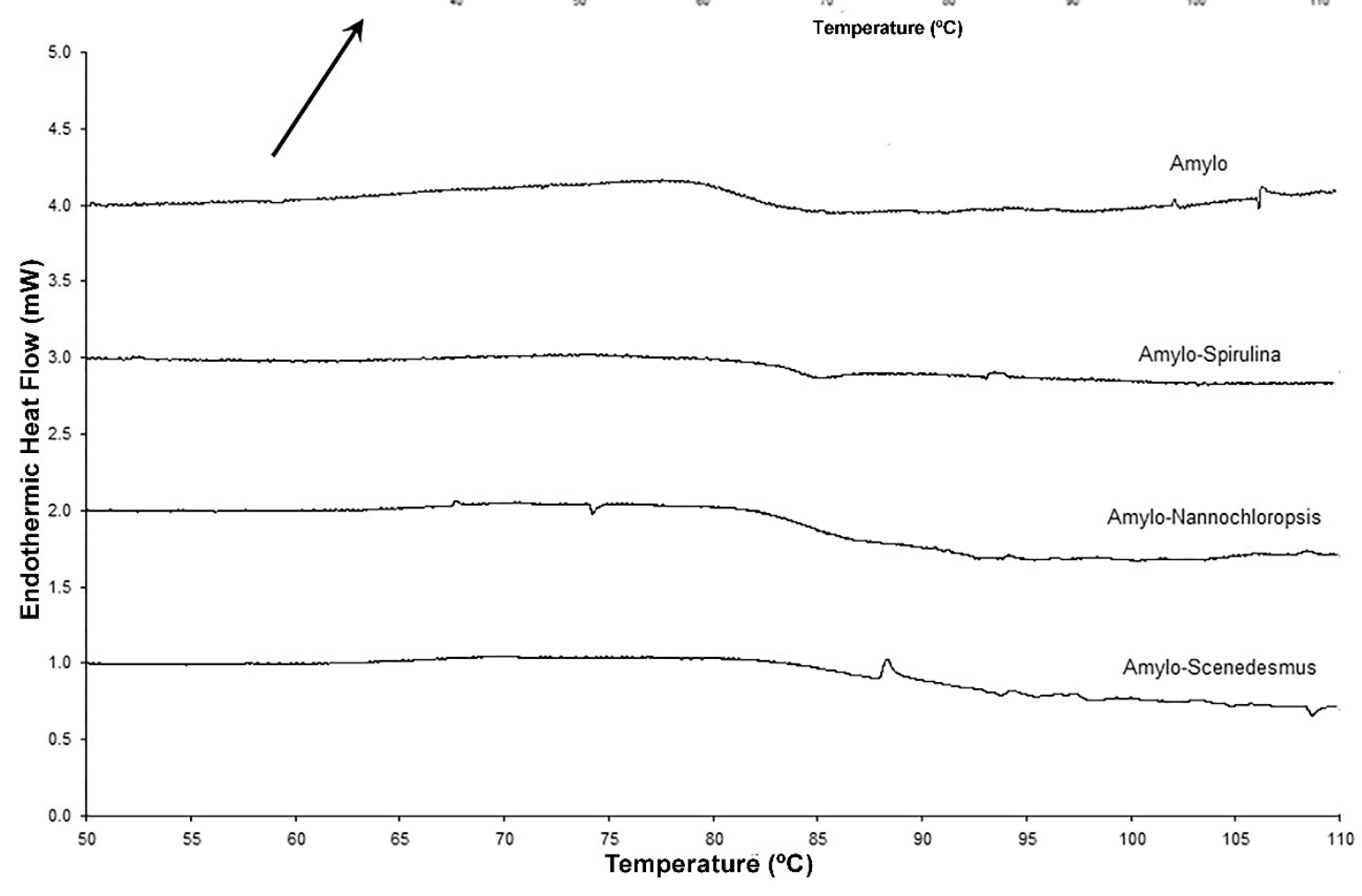

251

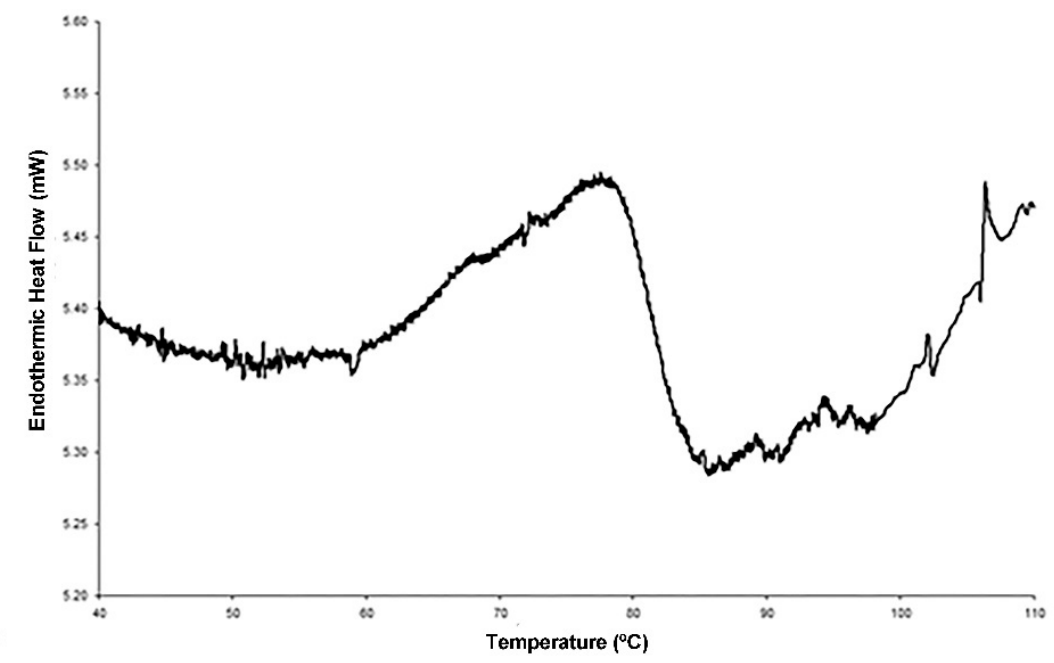

252 Figure 1. DSC thermograms from (A) corn starch samples and (B) high amylose starch 253 samples. Curves have been offset for clarity.

254

255 Table 1. Onset $\left(\mathrm{T}_{\mathrm{O}}\right)$, peak maximum $\left(\mathrm{T}_{\mathrm{P}}\right)$ and conclusion $\left(\mathrm{T}_{\mathrm{C}}\right)$ temperatures, entalphy $(\Delta \mathrm{H})$ 256 and peak height index (PHI) values for the gelatinisation endotherm of pure starch and 257 starch-microalgae suspensions. Entalphy values are expressed in J/g of dry starch. Standard 258 deviation values on the last digit are shown in parantheses.

\begin{tabular}{|l|l|l|l|l|}
\hline $\mathrm{T}_{\mathrm{O}}\left({ }^{\circ} \mathrm{C}\right)$ & $\mathrm{T}_{\mathrm{P}}\left({ }^{\circ} \mathrm{C}\right)$ & $\mathrm{T}_{\mathrm{C}}\left({ }^{\circ} \mathrm{C}\right)$ & $\Delta \mathrm{H}(\mathrm{J} / \mathrm{g})$ & $\mathrm{PHI}$ \\
\hline
\end{tabular}




\begin{tabular}{|l|c|c|c|c|c|}
\hline Corn & $62(1)$ & $64(2)$ & $67(1)$ & $10(6)$ & 5.0 \\
\hline Corn-Spirulina & $62(1)$ & $64(1)$ & $69(1)$ & $9(1)$ & 4.5 \\
\hline Corn-Nannochloropsis & $62.2(8)$ & $65.3(5)$ & $68.3(1)$ & $10.8(8)$ & 3.5 \\
\hline Corn-Scenedesmus & $61.2(2)$ & $64.0(1)$ & $66(2)$ & $10.7(6)$ & 3.8 \\
\hline High Amylose & $73(1)$ & $77.7(8)$ & $85.4(5)$ & $13(6)$ & 2.8 \\
\hline High Amylose-Spirulina & $69(6)$ & $81(3)$ & $84(6)$ & $19(5)$ & 1.6 \\
\hline High Amylose-Nannochloropsis & $73(5)$ & $79.7(5)$ & $82(4)$ & $8(4)$ & 1.2 \\
\hline High Amylose-Scenedesmus & $77.4(5)$ & $80.5(7)$ & $88(4)$ & $22(2)$ & 7.1 \\
\hline
\end{tabular}

259

2603.2 Evolution of the starch crystalline and lamellar structure during the gelatinisation 261 process

262 To investigate the structural changes undergone by starch during the gelatinisation process, 263 the pure starch and starch-microalgae suspensions were characterised by means of 264 temperature-resolved simultaneous SAXS/WAXS experiments. While SAXS is useful to 265 characterise the lamellar structure of starch, WAXS provides information on the crystalline 266 structure.

267

\section{3.2.1. Crystalline structure}

269 The crystalline structure of the starch and starch-microalgae samples was investigated by 270 analyzing the WAXS patterns collected when heating the samples from $30^{\circ} \mathrm{C}$ up to $110^{\circ} \mathrm{C}$. 271 Figures 2 and 3 show the temperature-resolved WAXS curves (temperature steps of $10^{\circ} \mathrm{C}$ ) 272 for the corn and high amylose starch samples, respectively. As shown in Figure 2, all the 273 corn starch samples display typical A-type diffraction patterns, characterized by the main 274 diffraction peaks located at $2 \theta=15.0^{\circ}, 17.3^{\circ}, 18.0^{\circ}$ and $23.0^{\circ}$. Interestingly, a weak peak 275 located at ca. $20^{\circ}$, characteristic from the V-type crystalline structure (Amparo Lopez276 Rubio, Flanagan, Gilbert, et al., 2008), appears in the spectra of the corn starch loaded with 
277 Spirulina (pointed out by an arrow in Figure 2B). Similarly, V-type crystallites have been 278 reported to originate from the formation of complexes between the polar lipids found in 279 native starches and the amylose fraction (Zobel, 1988). In addition, weak but sharp peaks, 280 located at ca. $15.6^{\circ}, 20.3^{\circ}, 26.5^{\circ}, 29.2^{\circ}$ and $29.6^{\circ}$, are visible in the spectra of the sample 281 loaded with Spirulina and two peaks at $22.9^{\circ}$ and $29.6^{\circ}$ appear in the sample containing 282 Scenedesmus (indicated by discontinuous lines in Figures 2B and 2D). These peaks could 283 arise from crystalline components, such as fatty acids, which are known to be found in the 284 microalgae (Ahlgren, Gustafsson, \& Boberg, 1992; James, Al-Hinty, \& Salman, 1989; Ma, 285 et al., 2017; Matos, et al., 2016). In fact, as shown in Figure S1, the X-ray diffraction 286 patterns of the microalgae present several peaks, with the most intense ones located at $2 \theta$ 287 values of ca. $16.6^{\circ}, 20.3^{\circ}, 29.0^{\circ}, 30.8^{\circ}$ and $31.6^{\circ}$ for Spirulina, $27.3^{\circ}$ and $31.5^{\circ}$ for 288 Nannochloropsis and $18.5^{\circ}, 23.2^{\circ}$ and $29.5^{\circ}$ for Scenedesmus. The appearance of these 289 peaks in the WAXS patterns from the aqueous suspensions (even at $30^{\circ} \mathrm{C}$ ) is indicative of 290 the presence of crystallised lipids in the Spirulina and Scenedesmus microalgae. However, 291 only those lipids contained in the Spirulina microalgae seem to be able to establish strong 292 interactions with amylose, leading to the formation of V-type crystallites. The formation of 293 amylose-lipid complexes has been reported to depend on the chain size (with smaller lipid 294 molecules being more prone to form complexes (Cui \& Oates, 1999)) and the concentration 295 of the lipids (Tang \& Copeland, 2007). Wheat starch has been shown to form complexes 296 with stearic acid up to a certain lipid concentration, above which the X-ray diffraction peaks 297 characteristic of the V-type crystallites are accompanied by the appearance of additional 298 peaks arising from the presence of lipid aggregates (Tang \& Copeland, 2007). Thus, our 299 results seem to indicate that the concentration of lipids in the Spirulina microalgae is above 300 this critical point at which some of the lipids tend to self-associate. 
302 The estimated crystallinity index values for the samples at the initial temperature of $30^{\circ} \mathrm{C}$, 303 listed in Table 2, evidence a structural effect of the microalgae in decreasing the apparent 304 crystallinity of the starch granules, being this effect more obvious for the Nannochloropsis 305 species. Hydration has been shown to be a relevant factor affecting starch crystalline 306 structure, with a positive linear relationship between moisture content and crystallinity 307 index (Cheetham, et al., 1998; Qiao, et al., 2017). This is due to the fact that water favors 308 molecular motion and chain organization, thus promoting the formation of more ordered 309 structures (Qiao, et al., 2017). Therefore, the decreased apparent crystallinity observed for 310 the starch-microalgae suspensions is most likely a consequence of a limited moisture 311 accessibility towards the interior of starch granules, i.e. a certain amount of the added water 312 is taken up by the microalgae. The WAXS apparent crystallinity drop is in contrast with the 313 DSC results, which show that the gelatinisation enthalpy of corn starch is unaffected by the 314 presence of microalgae. The discrepancy between DSC and WAXS arises from (i) the lower 315 sensitivity of DSC to determine crystalline transitions as compared to WAXS and (ii) the 316 susceptibility of these techniques to identify crystalline or molecular order. With regards to 317 (i), it has been shown that X-ray scattering is indeed able to detect residual crystallinity at 318 temperatures higher than those at which the DSC gelatinisation endotherm is completed 319 (Wang, Zhang, Wang, \& Copeland, 2016). Furthermore, in relation to (ii), whereas the 320 thermal transition determined by DSC accounts for the dissociation and unwinding of 321 helical structures (amylopectin double helices, amylose-lipid complexes and V-type single 322 helices), WAXS is only susceptible to those helical structures which are packed forming 323 crystallites. Thus, the presence of microalgae does not perturb the molecular order (i.e. 324 amount of helical structures) but restrains water access towards the interior of the granules, 325 hindering the organisation of these helices into crystallites and hence, reducing the apparent 326 crystallinity estimated by WAXS. The temperature range at which the crystallinity starts 
327 decreasing for all the samples, i.e. $60-70^{\circ} \mathrm{C}$ (cf. Figure 4A), is well correlated to the 328 gelatinisation temperature range determined from the DSC characterization (cf. Table 1).

329
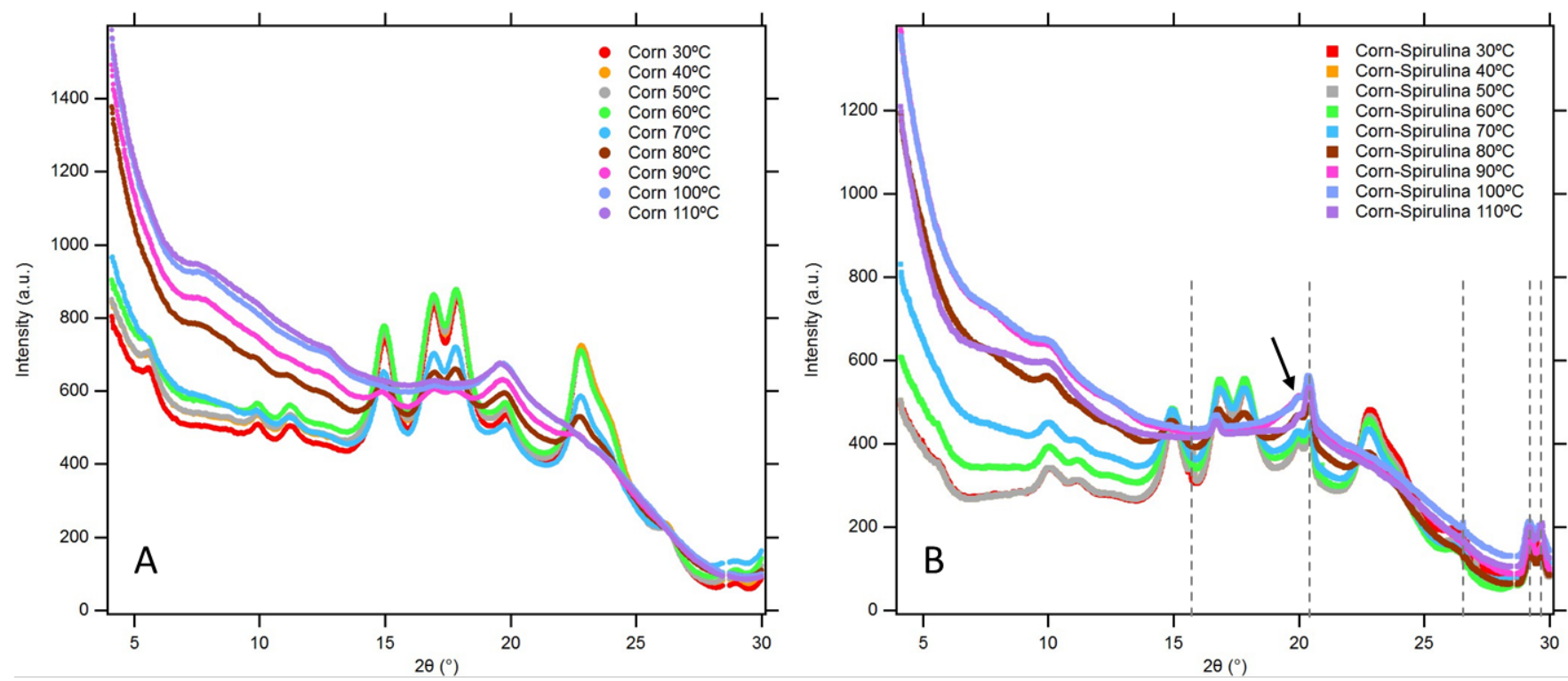

330
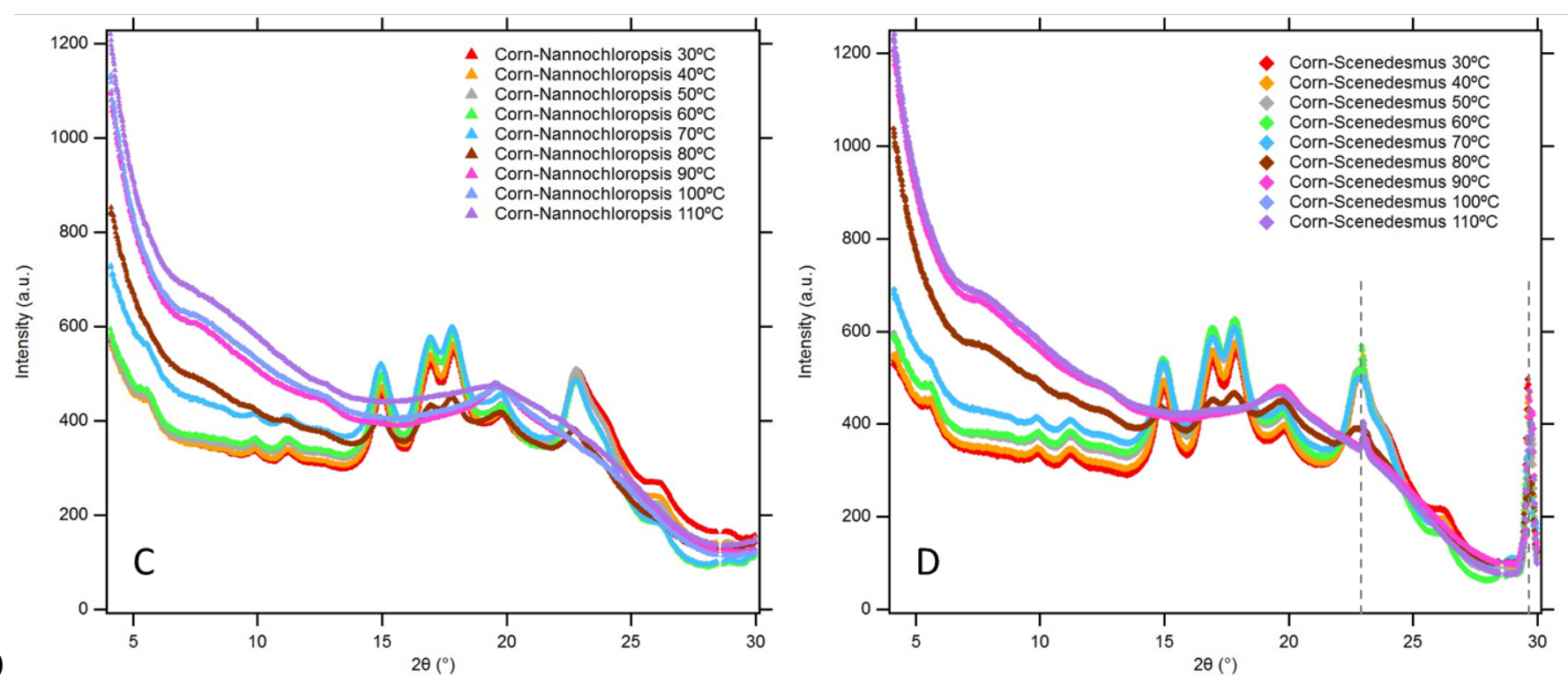

331 Figure 2. Temperature-resolved WAXS patterns of corn starch samples. (A) pure corn

332 starch, (B) corn starch-Spirulina, (C) corn starch-Nannochloropsis and (D) corn starch-

\section{Scenedesmus.}

334

335 As shown in Figure 3, the WAXS patterns from the high amylose corn starch samples differ

336 significantly from the corn starch patterns, with peak positions characteristic of the B-type 
337 crystalline allomorph. A transition from A-type to B-type crystallinity has been previously 338 reported for corn starches when increasing the amylose content across a range of $0-84 \%$ 339 (Cheetham, et al., 1998). This is a consequence of longer amylopectin helical chains found 340 in high amylose starches, which are preferentially crystallised into the B-type allomorph 341 (Gernat, Radosta, Anger, \& Damaschun, 1993). As observed for the corn starch, the peak 342 indicative of V-type crystallinity is detected in the sample containing Spirulina and sharp 343 peaks are visible in the scattering patterns of high amylose starch with Spirulina (peaks 344 located at ca. $15.0^{\circ}, 20.4^{\circ}, 26.6^{\circ}$ and $29.2^{\circ}$ ) and Scenedesmus (peaks located at ca. $23.0^{\circ}$ 345 and $29.7^{\circ}$ ). The formation of V-type crystallites as a result of amylose-lipid complexes may 346 have important implications not only for the industrial processing of starch-based products, 347 but also from the nutritional perspective. For instance, it has been reported that when 348 amylose is complexed with lipids it is hydrolyzed and absorbed in the gastrointestinal tract 349 to the same extent as free amylose but at a slower rate (J. Holm, et al., 1983). Additionally, 350 the V-type amylose-lipid complexes have been shown to produce a decrease in the amylose 351 solubility, hence increasing the gelatinisation temperature (Eliasson, Carlson, \& Larsson, 352 1981) and postpone retrogradation, thus enabling longer storage times (Krog, 1971). It 353 should be mentioned that the fact that a strong impact on the gelatinisation temperature is 354 not clearly observed in this work is most likely related to the low microalgae loadings added 355 into the starch aqueous suspensions (only 0.8 wt.-\% with regards to the starch weight). The 356 calculated crystallinity values at $30^{\circ} \mathrm{C}$ are $21.5 \%, 26.8 \%, 13.9 \%$ and $17.9 \%$ for the high 357 amylose, high amylose-Spirulina, high amylose-Nannochloropsis and high amylose358 Scenedesmus samples, respectively. It is also worth noting that, as seen in Figure 4B, the 359 crystallinity of all the samples decreases significantly within the temperature range of ca. $36070-90^{\circ} \mathrm{C}$, which is in agreement with the temperature range determined from the DSC 361 gelatinisation endotherms. However, it should be noted that, in agreement with previous 
362 studies (Wang, et al., 2016), the samples still present residual structural order at 363 temperatures higher than the DSC gelatinisation endotherm end temperature. It is also 364 worth noting that even though at the final temperature of $110^{\circ} \mathrm{C}$ the starch crystalline 365 structure has been almost completely destroyed, the peaks characteristic of the crystallised 366 lipids present in the Spirulina and Scenedesmus microalgae are still detected, indicating the 367 high thermal stability of these compounds.

368

369 The fact that the corn and high amylose starch present similar crystallinity values contrasts 370 with previous works stating that the crystallinity index is negatively correlated with the 371 amylose content (Gernat, et al., 1993). This may be true for low to intermediate amylose 372 contents, where the A-type allomorph is preserved; however, it could be hypothesised that 373 the longer helical structures present in starches with greater amylose contents and which 374 are crystallised into the B-type conformation, do not significantly affect the overall 375 crystallinity index. In fact, it has been shown that increasing the amylose content in B-type 376 starch does not have a strong impact on the crystallinity as it does in A-type starch 377 (Cheetham, et al., 1998). The complexation of amylose with the lipids from the added 378 microalgae seems to have a positive impact on the starch crystallinity. Thus, while the 379 general trend when incorporating microalgae into the suspensions is a reduction in the 380 apparent crystallinity (especially in the case of the Nannochloropsis) due to a reduced 381 swelling of the starch granules, this effect is reverted in the case of the high amylose starch 382 loaded with Spirulina, as in this case a greater amount of lipid-amylose complexes are 383 formed. 

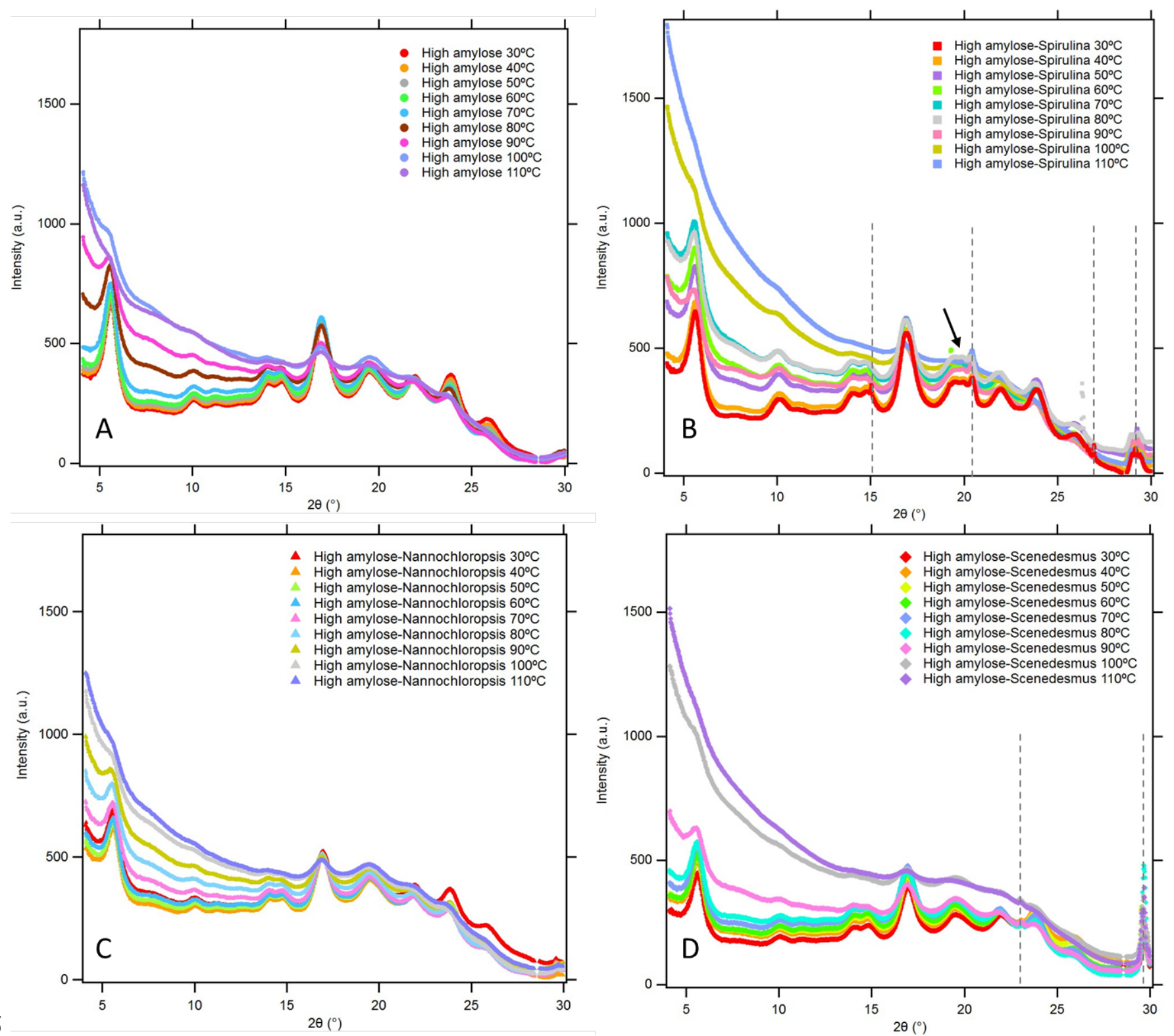

386 Figure 3. Temperature-resolved WAXS patterns of high amylose starch samples. (A) pure

387 high amylose starch, (B) high amylose starch-Spirulina, (C) high amylose starch388 Nannochloropsis and (D) high amylose starch-Scenedesmus.

390 Table 2. Lamellar peak parameters and crystallinity $\left(\mathrm{X}_{\mathrm{C}}\right)$ for the native samples $\left(\mathrm{T}=30^{\circ} \mathrm{C}\right)$.

391 The lamellar peak parameters were obtained by fitting the experimental SAXS data to the 392 sum function of a power-law plus a Gaussian-Lorentzian peak and crystallinity was obtained 393 from the peak fitting of the WAXS patterns. Standard deviation values on the last digit are 394 shown in parentheses. 


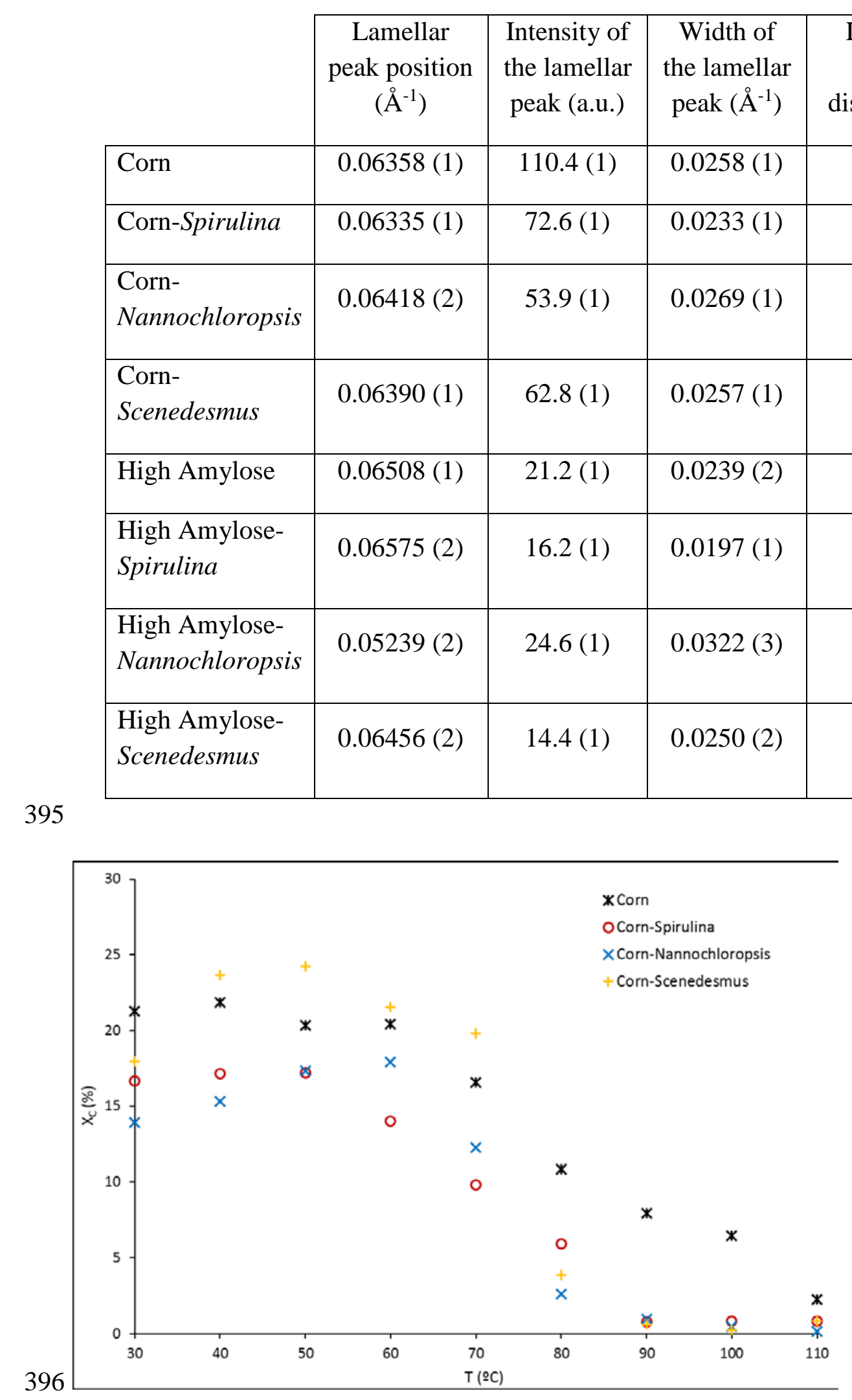




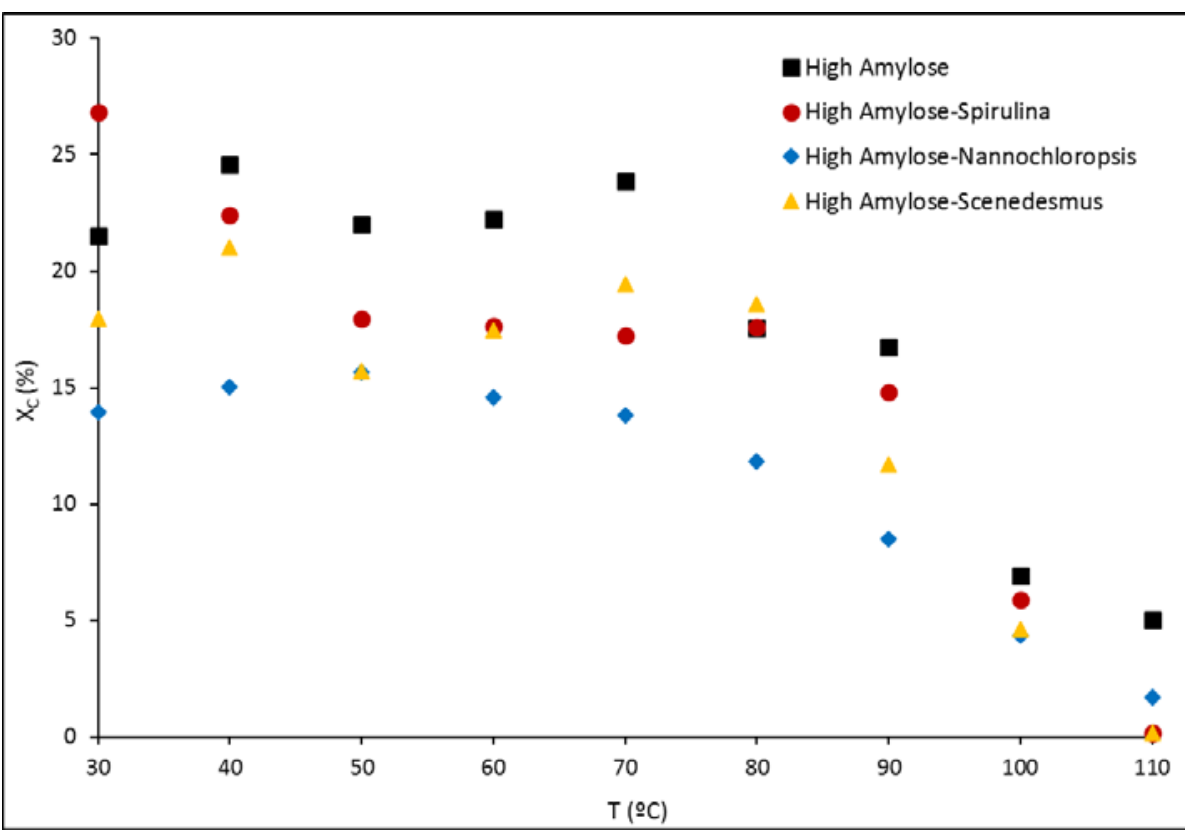

398 Figure 4. Evolution of the crystallinity index for the corn starch (A) and high amylose starch 399 (B) samples.

400

\section{3.2.2. Lamellar structure}

402 The crystalline regions constituted by the tightly packed helical structures are alternated with 403 amorphous regions, forming the next structural level known as lamellae. Due to their 404 different physical density, the crystalline and amorphous domains present distinct X-ray 405 scattering length density and, thus, the presence of these lamellar structures gives rise to the 406 appearance of scattering features in the SAXS patterns of starch samples. To investigate the 407 structural changes taking place in the lamellar arrangement during the gelatinisation process, 408 as well as the effect of the microalgae addition, the SAXS patterns were collected 409 simultaneously to the WAXS patterns when heating up the samples. As shown in Figure 5, 410 at the initial temperature of $30^{\circ} \mathrm{C}$ all the samples present a well-defined peak located at ca. $4110.06 \AA^{-1}$, which corresponds to the lamellar peak characteristic of hydrated native starch 412 (Blazek \& Gilbert, 2011; Chanvrier, et al., 2007; Salman, et al., 2009). The position of this 413 peak is related to the thickness of crystalline and amorphous lamellae. Additionally, the peak 
414 area depends on the scattering length density difference between the crystalline and 415 amorphous domains. To extract detailed structural information from the obtained SAXS 416 patterns, the experimental data within the $\mathrm{q}$ range of $0.045-0.2 \AA^{-1}$ were fitted using a 417 mathematical function based on the sum of a power-law plus a Lorentzian-Gaussian peak 418 (cf. section 2.5) and the main parameters extracted from the fits are gathered in Table 2. The 419 first observation is that while the lamellar distance is not strongly affected by the starch 420 amylose content, remaining within the range of 9-10 $\AA$ typically reported for native starches 421 (P. J. Jenkins \& Donald, 1995), the intensity of the lamellar peak is greatly reduced for the 422 high amylose starch. This is probably originated by a lower scattering length density contrast 423 in this sample. In fact, it has been reported that while the lamellar distance remains constant, 424 the size of the crystalline regions increases and the electron density difference between the 425 crystalline and amorphous regions decreases with the amylose content (P. J. Jenkins, et al., 426 1995). This is a consequence of the larger amylopectin chain length observed for high 427 amylose starches, which results in the formation of the less densely packed B-type 428 crystallites. A-type structures are closely packed with water molecules between each double 429 helical structure, whereas B-type are more open and water molecules are located in the 430 central cavity formed by six double helices (Imberty, Buléon, Tran, \& Pérez, 1991). Thus, 431 in low amylose starches (with short amylopectin chains which are crystallised into the A432 type structure) the crystalline lamellae are smaller but more densely packed, whereas in high 433 amylose starches (with B-type crystallites formed by large amylopectin chains) crystalline 434 lamellae become larger but more loosely packed.

435

436 As clearly observed in Figure 5 and confirmed by the fitting parameters, the incorporation 437 of microalgae into the corn starch suspensions leads to a marked decrease in the relative 438 intensity of the lamellar peak. Conversely, the peak position and width are not significantly 
439 affected. This indicates that the addition of microalgae reduces the scattering length density 440 contrast but does not affect the lamellae structural parameters. Together with the DSC and 441 WAXS characterisation, this suggests that the presence of microalgae does not affect the 442 molecular order but impairs the organisation of these helical structures into densely packed 443 and ordered crystalline domains by limiting to some extent the swelling of starch granules. 444 The reduced apparent crystallinity would then contribute to the observed decrease in the 445 scattering length density contrast. In the case of the high amylose starch, the presence of 446 microalgae does not have a strong effect on the lamellar peak intensity, but the peak position 447 and width are affected differently depending on the microalgae species. A significantly 448 broader lamellar peak, with a greater associated lamellar repeat distance of ca. $12 \AA$ is 449 observed upon incorporation of Nannochloropsis, being indicative of more heterogeneous 450 lamellae in the presence of this microalgae species. This effect can also be explained by a 451 decreased water accessibility towards the interior of starch granules, since it has been 452 reported that in the dry state only a small fraction of starch helices can be aligned and packed 453 to construct the semi-crystalline lamellar structure (Qiao, et al., 2017).Together with the 454 reduced crystallinity estimated from the WAXS patterns, this result indicates that the 455 presence of Nannochloropsis has a strong impact on the swelling mechanism of starch 456 granules. In contrast, the addition of Spirulina has the opposite effect, i.e. a greater degree 457 of crystalline order is attained (as indicated by the higher crystallinity index) and more 458 homogeneous lamellae are formed (as suggested by the reduced lamellar peak width). The 459 distinct effect of these microalgae on the starch structure and the gelatinisation process may 460 be explained based on their different composition and, mostly, on their distinct cell wall 461 structure. The total lipidic contents have been reported as ca. 6\% for Spirulina (Matos, et al., 462 2016), 8-16\% for Nannochloropsis (Matos, et al., 2016) and up to 30\% for Scenedesmus 463 microalgae (Jiang, et al., 2017; Ma, et al., 2017). However, the Nannochloropsis microalgae 
464 have been shown to possess a tough cell wall, formed by a cellulosic inner layer and an outer 465 layer rich in algaenans, which requires intense sonication treatments to be disrupted (Fabra, 466 et al., 2017; Scholz, et al., 2014). The existence of such resistant cell walls would then 467 impede the release of lipids from the cells towards the aqueous medium. The cell wall 468 structure of the Scenedesmus species also contains an inner cellulosic layer, which is then 469 covered by a thin middle layer and an outer pectic layer (Bisalputra \& Weier, 1963). On the 470 other hand, the cell walls in Spirulina seem to be richer in proteins and they present porous 471 features which may facilitate the release of cell components (Van Eykelenburg, 1977). To 472 corroborate their different cell wall integrity, raw microalgae were characterised by TEM 473 and the crystalline components in their cell walls were stained with uranyl acetate. As 474 observed in Figure S2, while the Nannochloropsis microalgae presented intact cell walls, 475 intensely stained and with clearly defined edges, some of the cell walls in Spirulina 476 microalgae seemed to be disrupted as a result of the sample preparation process. Therefore, 477 despite the lower lipidic content of Spirulina, these components could more easily be 478 released through the weak cell walls, being then able to interact with the amylose fraction in 479 starch and forming complexes which induce a higher degree of crystallinity, as well as 480 promoting the formation of more homogeneous lamellae. 


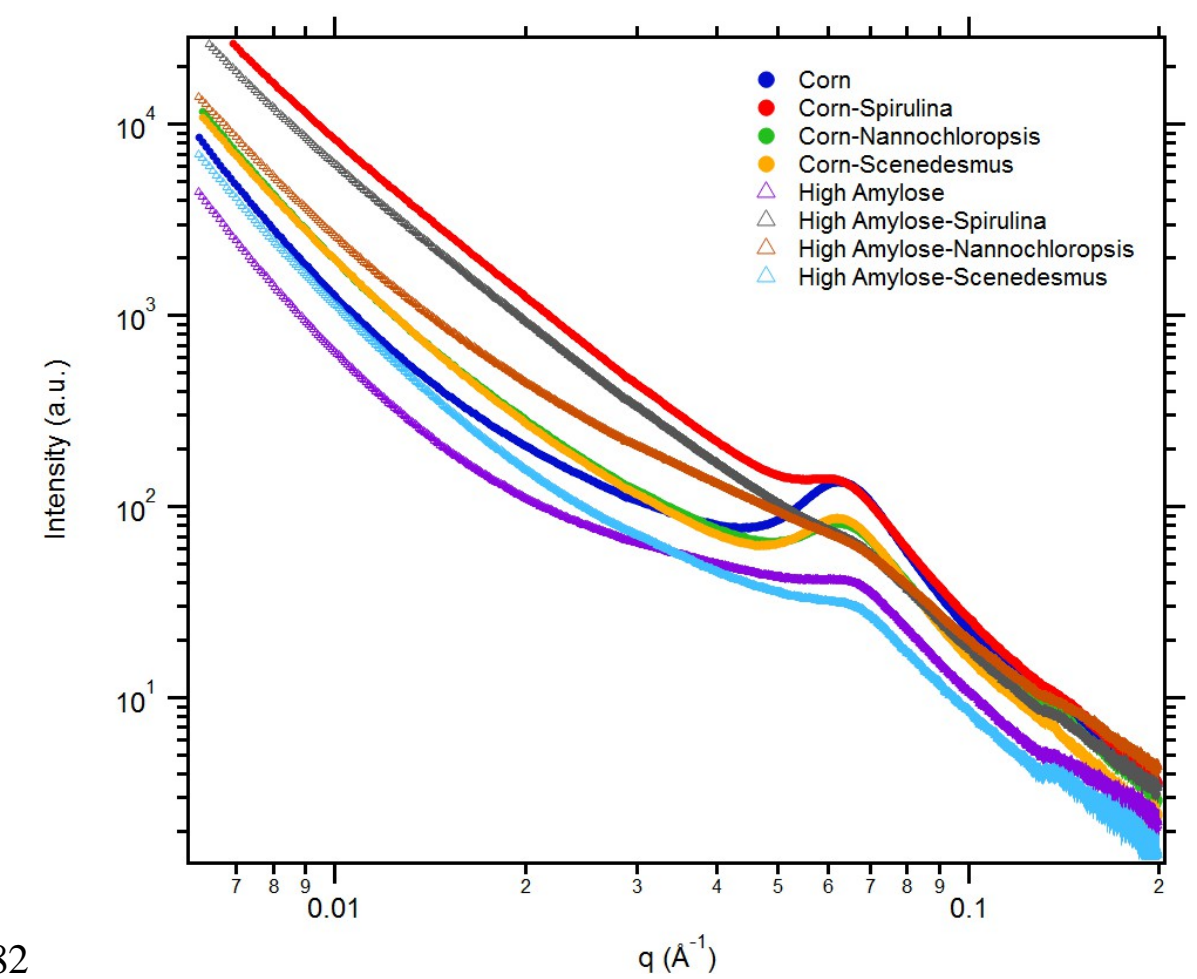

483 Figure 5. SAXS patterns of starch and starch-microalgae suspensions in water at $30^{\circ} \mathrm{C}$.

484

485 The structural changes undergone by starch during the gelatinisation process were studied 486 by heating the prepared suspensions up to $110^{\circ} \mathrm{C}$ and recording the SAXS patterns. The 487 results for the pure corn starch and microalgae-loaded samples are shown in Figure 6. As 488 observed from this figure, the relative intensity of the lamellar peak decreases gradually 489 when the temperature increases, reaching a point at which the well-resolved peak is replaced 490 by a very broad shoulder, which in some of the samples is eventually lost at the highest 491 temperatures. A similar broad shoulder has been observed for retrograded starch and it has 492 been correlated with the growth of crystals in an amorphous matrix (Amparo Lopez-Rubio, 493 Flanagan, Shrestha, Gidley, \& Gilbert, 2008). The distortion of the lamellar structure is 494 evidenced by a strong increase in the width of the lamellar peak and in the associated lamellar 495 repeating distance. As deduced from Figure S3, which shows the evolution of the calculated 496 lamellar repeat distance as a function of the temperature, this transition takes place within 497 the temperature range of $80-100^{\circ} \mathrm{C}$. 

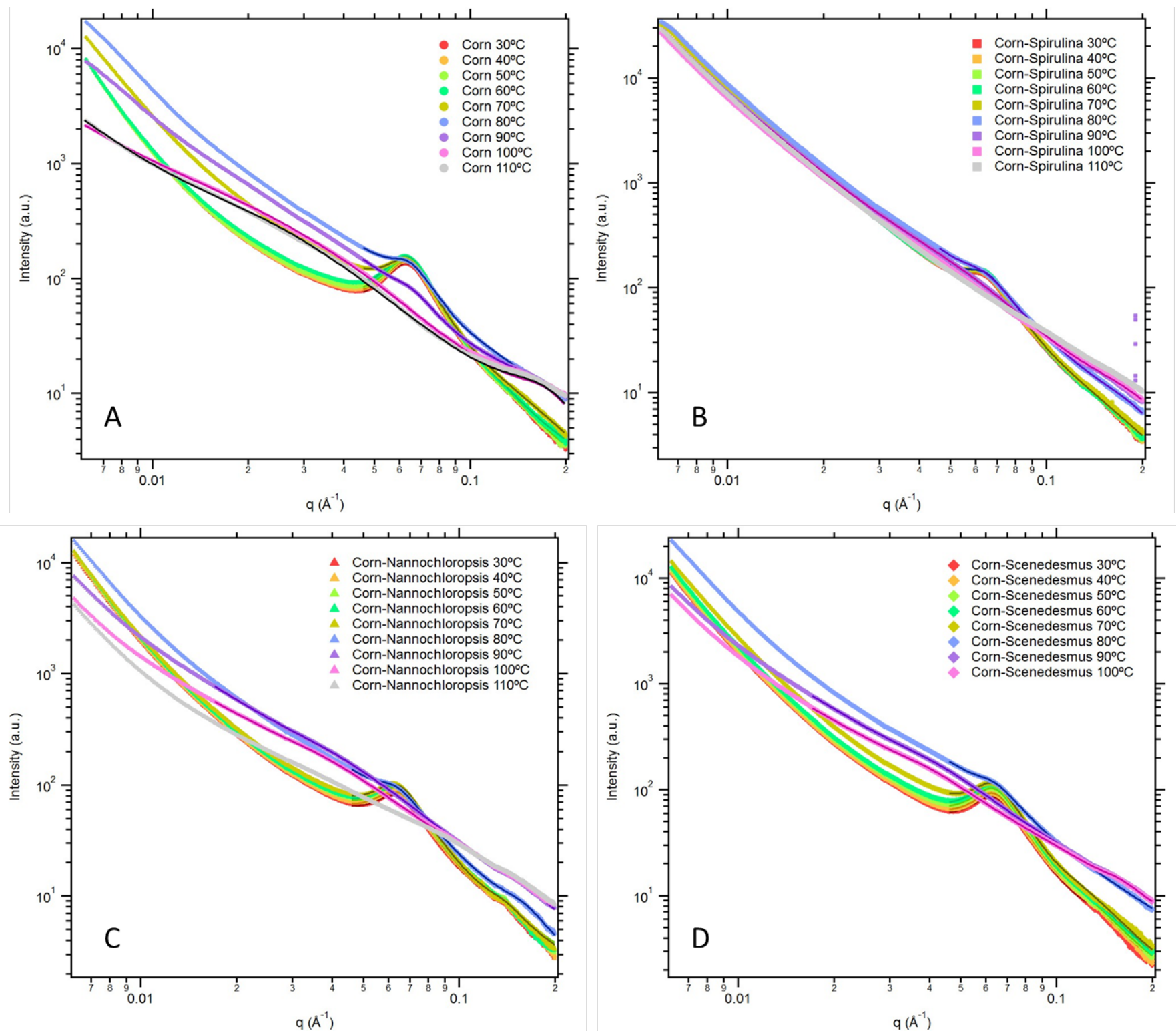

501 Figure 6. Temperature-resolved SAXS patterns of corn starch samples and the

502 corresponding fitting curves. (A) pure corn starch, (B) corn starch-Spirulina, (C) corn starch-

$503 \quad$ Nannochloropsis and (D) corn starch-Scenedesmus.

505 As shown in Figure 7, increasing the temperature has a similar effect on the lamellar peak in

506 the high amylose starch samples. In that case, the transition from the lamellar peak to the

507 broad shoulder also takes place within a temperature range of $80-100^{\circ} \mathrm{C}$ for the pure and 
508 microalgae-loaded samples (cf. Figure S1). As opposed to the corn starch samples, the

509 shoulder is still visible for all the high amylose samples at the final temperature of $110^{\circ} \mathrm{C}$,

510 indicating that starch still presents a residual long-range structure. This, together with the

511 residual crystallinity detected in the WAXS patterns when raising the temperature above the

512 gelatinisation endotherm end temperatures calculated from DSC, supports the hypothesis

513 presented by Wang et al. that the gelatinization endotherm of starch does not represent

514 complete starch gelatinization (Wang, et al., 2016).

515
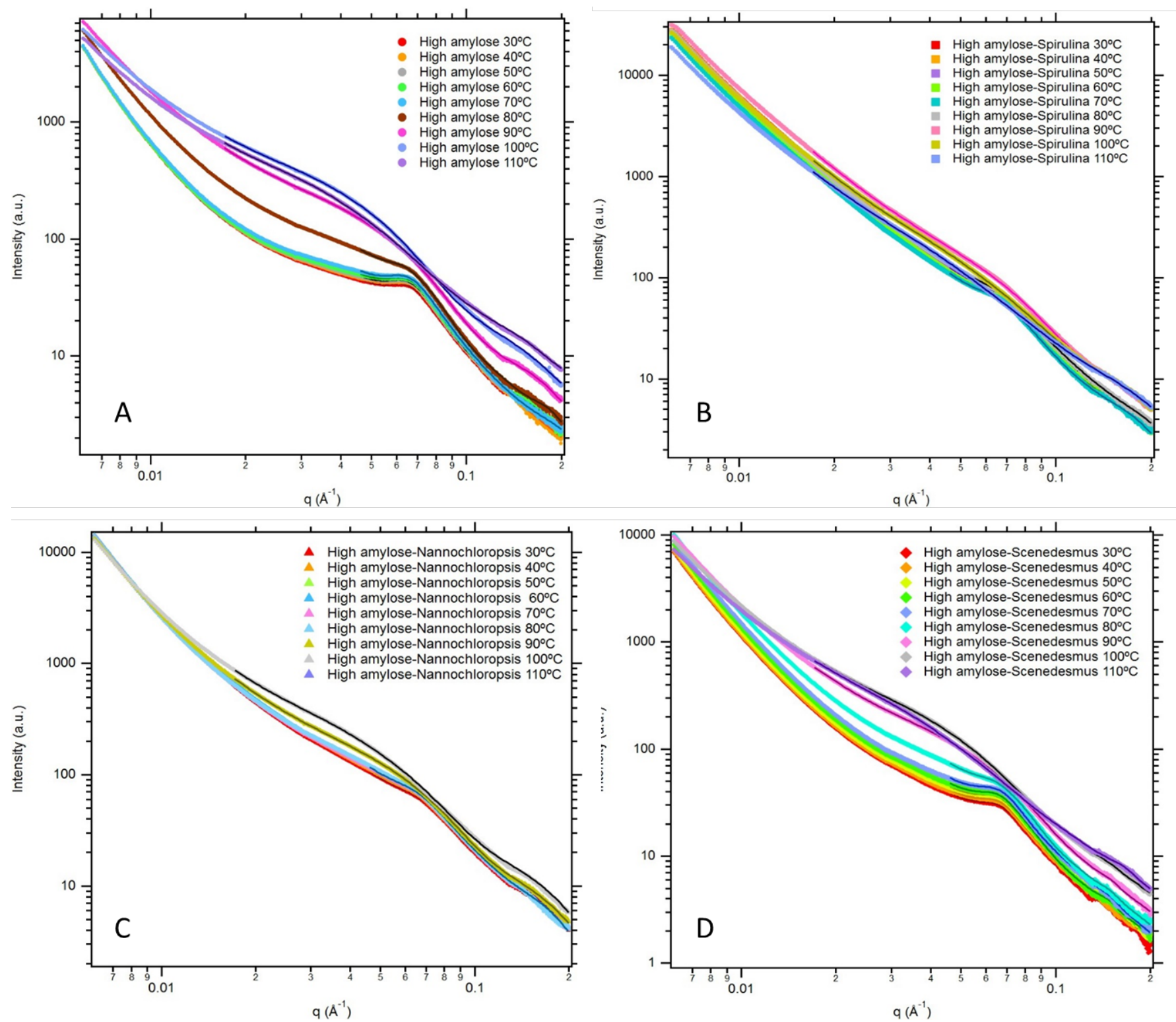
$517 \quad$ Figure 7. Temperature-resolved SAXS patterns of high amylose starch samples and the 518 corresponding fitting curves. (A) pure high amylose starch, (B) high amylose starch519 Spirulina, (C) high amylose starch-Nannochloropsis and (D) high amylose starch$520 \quad$ Scenedesmus.

521

522 4. Conclusions 
523 This study presents a detailed characterisation of the structural effects undergone by two

524 types of starch with different amylose contents during the gelatinisation process as affected

525 by the addition of different microalgae, carried out by means of temperature-resolved

526 simultaneous SAXS/WAXS experiments, combined with DSC analyses.

527 The three different microalgae species incorporated into the starch aqueous suspensions,

528 namely Spirulina, Nannochloropsis and Scenedesmus, have shown to induce structural

529 changes during the gelatinisation process. Interestingly, the observed structural effects are

530 strongly dependent on the microalgae cell wall structure and integrity. In general, the

531 presence of microalgae limits water accessibility towards the interior of starch granules,

532 hence impeding the organisation of amylopectin double helices into highly ordered

533 crystalline regions, leading to the formation of more heterogeneous lamellar structures with

534 decreased apparent crystallinity. This effect is more evident in the case of the

535 Nannochloropsis species, whose resistant cell wall remains intact and does not allow the

536 release of any component towards the aqueous medium. In contrast, the weak cell walls in

537 the Spirulina microalgae are easily disrupted, enabling the release of the lipids contained

538 within the cells. These free lipids form helical inclusion complexes with the starch amylose

539 chains, promoting the formation of a certain fraction of V-type crystallites, increasing the

540 overall crystalline index and resulting in the formation of more homogeneous lamellar

541 structures. The formation of these lipid-amylose complexes is relevant from the processing

542 and nutritional point of view. Starch granules become more thermodynamically stable, i.e.

543 the gelatinisation temperature increases, and the complexed amylose is known to be more

544 slowly digested in the gastrointestinal tract than free amylose. 
These results support the potential of scattering techniques in providing a detailed structural information for starch samples, being able to simulate industrial-relevant processes such as the gelatinisation phenomenon by means of temperature-resolved experiments. The incorporation of microalgae with permeable and/or weak cell walls into starch may be interesting to generate more crystalline and thermodynamically stable products through the formation of lipid-amylose complexes.

\section{Acknowledgements}

Synchrotron experiments were performed at NCD beamline at ALBA Synchrotron with the collaboration of ALBA staff (2016021658 project). M.J. Fabra, Marta Martinez-Sanz and L.G. Gómez-Mascaraque are recipients of a Ramon y Cajal (RYC-2014-158), Juan de la Cierva (IJCI-2015-23389) and predoctoral (call 2013) contracts from the Spanish Ministry of Economy, Industry and Competitiveness, respectively.

\section{References}

Ahlgren, G., Gustafsson, I. B., \& Boberg, M. (1992). Fatty acid content and chemical composition of freshwater microalgae. Journal of phycology, 28(1), 37-50.

Biliaderis, C., Maurice, T., \& Vose, J. (1980). Starch gelatinization phenomena studied by differential scanning calorimetry. Journal of Food Science, 45(6), 1669-1674.

Bisalputra, T., \& Weier, T. E. (1963). The Cell Wall of Scenedesmus quadricauda. American Journal of Botany, 50(10), 1011-1019.

Blazek, J., \& Gilbert, E. P. (2011). Application of small-angle X-ray and neutron scattering techniques to the characterisation of starch structure: A review. Carbohydrate Polymers, 85(2), 281-293.

Chanvrier, H., Uthayakumaran, S., Appelqvist, I. A. M., Gidley, M. J., Gilbert, E. P., \& López-Rubio, A. (2007). Influence of Storage Conditions on the Structure, Thermal Behavior, and Formation of Enzyme-Resistant Starch in Extruded Starches. Journal of Agricultural and Food Chemistry, 55(24), 9883-9890.

Cheetham, N. W. H., \& Tao, L. (1998). Variation in crystalline type with amylose content in maize starch granules: an X-ray powder diffraction study. Carbohydrate Polymers, 36(4), 277-284.

Cui, R., \& Oates, C. G. (1999). +The effect of amylose-lipid complex formation on enzyme susceptibility of sago starch. Food Chemistry, 65(4), 417-425. 
De Marco, E. R., Steffolani, M. E., Martínez, C. S., \& León, A. E. (2014). Effects of spirulina biomass on the technological and nutritional quality of bread wheat pasta. LWT-Food Science and Technology, 58(1), 102-108.

Draaisma, R. B., Wijffels, R. H., Slegers, P. M., Brentner, L. B., Roy, A., \& Barbosa, M. J. (2013). Food commodities from microalgae. Current Opinion in Biotechnology, 24(2), 169-177.

Eliasson, A. C., Carlson, T. G., \& Larsson, K. (1981). Some effects of starch lipids on the thermal and rheological properties of wheat starch. Starch-Stärke, 33(4), 130134.

Fabra, M. J., Martínez-Sanz, M., Gomez-Mascaraque, L. G., Coll, J. M., Martínez, J. C., \& Lopez-Rubio, A. (2017). Development and characterization of hybrid corn starch-microalgae films: effect of US pre-treatment on structural, barrier and mechanical performance. Carbohydrate Polymers, under review.

Fradique, M., Batista, A. P., Nunes, M. C., Gouveia, L., Bandarra, N. M., \& Raymundo, A. (2010). Incorporation of Chlorella vulgaris and Spirulina maxima biomass in pasta products. Part 1: Preparation and evaluation. Journal of the Science of Food and Agriculture, 90(10), 1656-1664.

Fradique, M., Batista, A. P., Nunes, M. C., Gouveia, L., Bandarra, N. M., \& Raymundo, A. (2013). Isochrysis galbana and Diacronema vlkianum biomass incorporation in pasta products as PUFA's source. LWT-Food Science and Technology, 50(1), 312-319.

Gernat, C., Radosta, S., Anger, H., \& Damaschun, G. (1993). Crystalline Parts of Three Different Conformations Detected in Native and Enzymatically Degraded Starches. Starch - Stärke, 45(9), 309-314.

Hermansson, A.-M., \& Svegmark, K. (1996). Developments in the understanding of starch functionality. Trends in Food Science \& Technology, 7(11), 345-353.

Holm, J., Björck, I., Ostrowska, S., Eliasson, A. C., Asp, N. G., Larsson, K., \& Lundquist, I. (1983). Digestibility of Amylose-Lipid Complexes in-vitro and in-vivo. Starch - Stärke, 35(9), 294-297.

Holm, J., Lundquist, I., Björck, I., Eliasson, A.-C., \& Asp, N.-G. (1988). Degree of starch gelatinization, digestion rate of starch in vitro, and metabolic response in rats. The American journal of clinical nutrition, 47(6), 1010-1016.

Hoover, R. (2001). Composition, molecular structure, and physicochemical properties of tuber and root starches: a review. Carbohydrate Polymers, 45(3), 253-267.

Ilavsky, J., \& Jemian, P. R. (2009). Irena: Tool suite for modeling and analysis of smallangle scattering. Journal of Applied Crystallography, 42(2), 347-353.

Imberty, A., Buléon, A., Tran, V., \& Pérez, S. (1991). Recent advances in knowledge of starch structure. Starch-Stärke, 43(10), 375-384.

Imberty, A., Chanzy, H., Pérez, S., Bulèon, A., \& Tran, V. (1988). The double-helical nature of the crystalline part of A-starch. Journal of Molecular Biology, 201(2), 365-378.

Imberty, A., \& Perez, S. (1988). A revisit to the three-dimensional structure of B-type starch. Biopolymers, 27(8), 1205-1221.

James, C. M., Al-Hinty, S., \& Salman, A. E. (1989). Growth and $\omega 3$ fatty acid and amino acid composition of microalgae under different temperature regimes. Aquaculture, 77(4), 337-351.

Jenkins, P. J., Cameron, R. E., \& Donald, A. M. (1993). A universal feature in the structure of starch granules from different botanical sources. Starch-Stärke, 45(12), 417420. 
Jenkins, P. J., \& Donald, A. M. (1995). The influence of amylose on starch granule structure. International Journal of Biological Macromolecules, 17(6), 315-321.

Jiang, L., Cheng, J., Pei, H., Zhang, S., Yang, Z., Nie, C., \& Zhang, L. (2017). Coupled microalgal cultivation with treatment of domestic sewage and high-level CO2. Environmental Technology(just-accepted), 1-20.

Kadam, S., \& Prabhasankar, P. (2010). Marine foods as functional ingredients in bakery and pasta products. Food Research International, 43(8), 1975-1980.

Kieffer, J., \& Wright, J. (2013). PyFAI: a Python library for high performance azimuthal integration on GPU. Powder Diffraction, 28(S2), S339-S350.

Kline, S. R. (2006). Reduction and analysis of SANS and USANS data using IGOR Pro. Journal of Applied Crystallography, 39(6), 895-900.

Krog, N. (1971). Amylose complexing effect of food grade emulsifiers. Starch-Stärke, 23(6), 206-210.

Krueger, B., Knutson, C., Inglett, G., \& Walker, C. (1987). A differential scanning calorimetry study on the effect of annealing on gelatinization behavior of corn starch. Journal of Food Science, 52(3), 715-718.

LeCorre, D., Bras, J., \& Dufresne, A. (2011). Influence of botanic origin and amylose content on the morphology of starch nanocrystals. Journal of Nanoparticle Research, 13(12), 7193-7208.

Li, M., Liu, P., Zou, W., Yu, L., Xie, F., Pu, H., Liu, H., \& Chen, L. (2011). Extrusion processing and characterization of edible starch films with different amylose contents. Journal of Food Engineering, 106(1), 95-101.

Liu, H., Xie, F., Yu, L., Chen, L., \& Li, L. (2009). Thermal processing of starch-based polymers. Progress in Polymer Science, 34(12), 1348-1368.

Liu, H., Yu, L., Xie, F., \& Chen, L. (2006). Gelatinization of cornstarch with different amylose/amylopectin content. Carbohydrate Polymers, 65(3), 357-363.

Lopez-Rubio, A., Flanagan, B. M., Gilbert, E. P., \& Gidley, M. J. (2008). A novel approach for calculating starch crystallinity and its correlation with double helix content: A combined XRD and NMR study. Biopolymers, 89(9), 761-768.

Lopez-Rubio, A., Flanagan, B. M., Shrestha, A. K., Gidley, M. J., \& Gilbert, E. P. (2008). Molecular Rearrangement Of Starch During In Vitro Digestion: Toward A Better Understanding Of Enzyme Resistant Starch Formation In Processed Starches. Biomacromolecules, 9(7), 1951-1958.

Lopez-Rubio, A., Htoon, A., \& Gilbert, E. P. (2007). Influence of extrusion and digestion on the nanostructure of high-amylose maize starch. Biomacromolecules, 8(5), 1564-1572.

Ma, C., Wen, H., Xing, D., Pei, X., Zhu, J., Ren, N., \& Liu, B. (2017). Molasses wastewater treatment and lipid production at low temperature conditions by a microalgal mutant Scenedesmus sp. Z-4. Biotechnology for Biofuels, 10(1), 111.

Mata, T. M., Martins, A. A., \& Caetano, N. S. (2010). Microalgae for biodiesel production and other applications: A review. Renewable and Sustainable Energy Reviews, 14(1), 217-232.

Matos, Â. P., Feller, R., Moecke, E. H. S., de Oliveira, J. V., Junior, A. F., Derner, R. B., \& Sant'Anna, E. S. (2016). Chemical Characterization of Six Microalgae with Potential Utility for Food Application. Journal of the American Oil Chemists' Society, 93(7), 963-972.

Matveev, Y. I., van Soest, J. J. G., Nieman, C., Wasserman, L. A., Protserov, V. A., Ezernitskaja, M., \& Yuryev, V. P. (2001). The relationship between 
thermodynamic and structural properties of low and high amylose maize starches. Carbohydrate Polymers, 44(2), 151-160.

Pérez, S., \& Bertoft, E. (2010). The molecular structures of starch components and their contribution to the architecture of starch granules: A comprehensive review. Starch-Stärke, 62(8), 389-420.

Qiao, D., Zhang, B., Huang, J., Xie, F., Wang, D. K., Jiang, F., Zhao, S., \& Zhu, J. (2017). Hydration-induced crystalline transformation of starch polymer under ambient conditions. International Journal of Biological Macromolecules.

Salman, H., Blazek, J., Lopez-Rubio, A., Gilbert, E. P., Hanley, T., \& Copeland, L. (2009). Structure-function relationships in A and B granules from wheat starches of similar amylose content. Carbohydrate Polymers, 75(3), 420-427.

Sarko, A., \& Wu, H. C. (1978). The Crystal Structures of A-, B-and C-Polymorphs of Amylose and Starch. Starch-Stärke, 30(3), 73-78.

Scholz, M. J., Weiss, T. L., Jinkerson, R. E., Jing, J., Roth, R., Goodenough, U., Posewitz, M. C., \& Gerken, H. G. (2014). Ultrastructure and composition of the Nannochloropsis gaditana cell wall. Eukaryotic cell, 13(11), 1450-1464.

Sevenou, O., Hill, S., Farhat, I., \& Mitchell, J. (2002). Organisation of the external region of the starch granule as determined by infrared spectroscopy. International Journal of Biological Macromolecules, 31(1), 79-85.

Spolaore, P., Joannis-Cassan, C., Duran, E., \& Isambert, A. (2006). Commercial applications of microalgae. Journal of Bioscience and Bioengineering, 101(2), 87-96.

Tang, M. C., \& Copeland, L. (2007). Analysis of complexes between lipids and wheat starch. Carbohydrate Polymers, 67(1), 80-85.

Tester, R. F., \& Morrison, W. R. (1990). Swelling and gelatinization of cereal starches. I. Effects of amylopectin, amylose, and lipids. Cereal Chem, 67(6), 551-557.

Van Eykelenburg, C. (1977). On the morphology and ultrastructure of the cell wall of Spirulina platensis. Antonie van Leeuwenhoek, 43(2), 89-99.

Varavinit, S., Shobsngob, S., Varanyanond, W., Chinachoti, P., \& Naivikul, O. (2003). Effect of amylose content on gelatinization, retrogradation and pasting properties of flours from different cultivars of Thai rice. Starch-Stärke, 55(9), 410-415.

Waigh, T. A., Gidley, M. J., Komanshek, B. U., \& Donald, A. M. (2000). The phase transformations in starch during gelatinisation: A liquid crystalline approach. Carbohydrate Research, 328(2), 165-176.

Wang, S., Zhang, X., Wang, S., \& Copeland, L. (2016). Changes of multi-scale structure during mimicked DSC heating reveal the nature of starch gelatinization. Scientific Reports, 6.

Yoshimura, M., Takaya, T., \& Nishinari, K. (1996). Effects of Konjac-Glucomannan on the Gelatinization and Retrogradation of Corn Starch As Determined by Rheology and Differential Scanning Calorimetry. Journal of Agricultural and Food Chemistry, 44(10), 2970-2976.

Yuryev, V. P., Kalistratova, E. N., van Soest, J. G., \& Niemann, C. (1998). Thermodynamic properties of barley starches with different amylose content. Starch-Stärke, 50(11-12), 463-466.

Zobel, H. F. (1988). Starch Crystal Transformations and Their Industrial Importance. Starch - Stärke, 40(1), 1-7. 
724 Supplementary Material

725

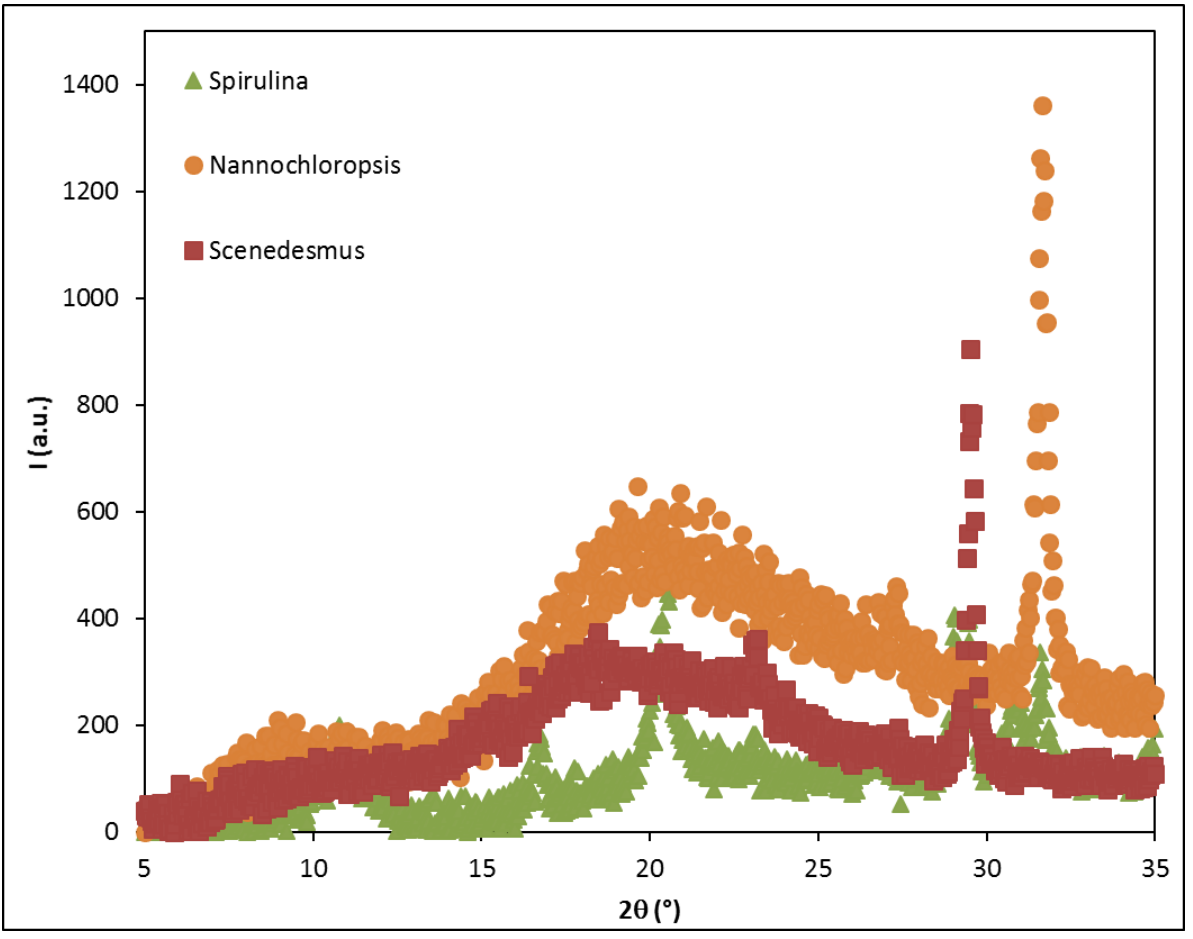

727 Figure S1. X-ray diffraction patterns of the raw microalgae (freeze-dried).

728

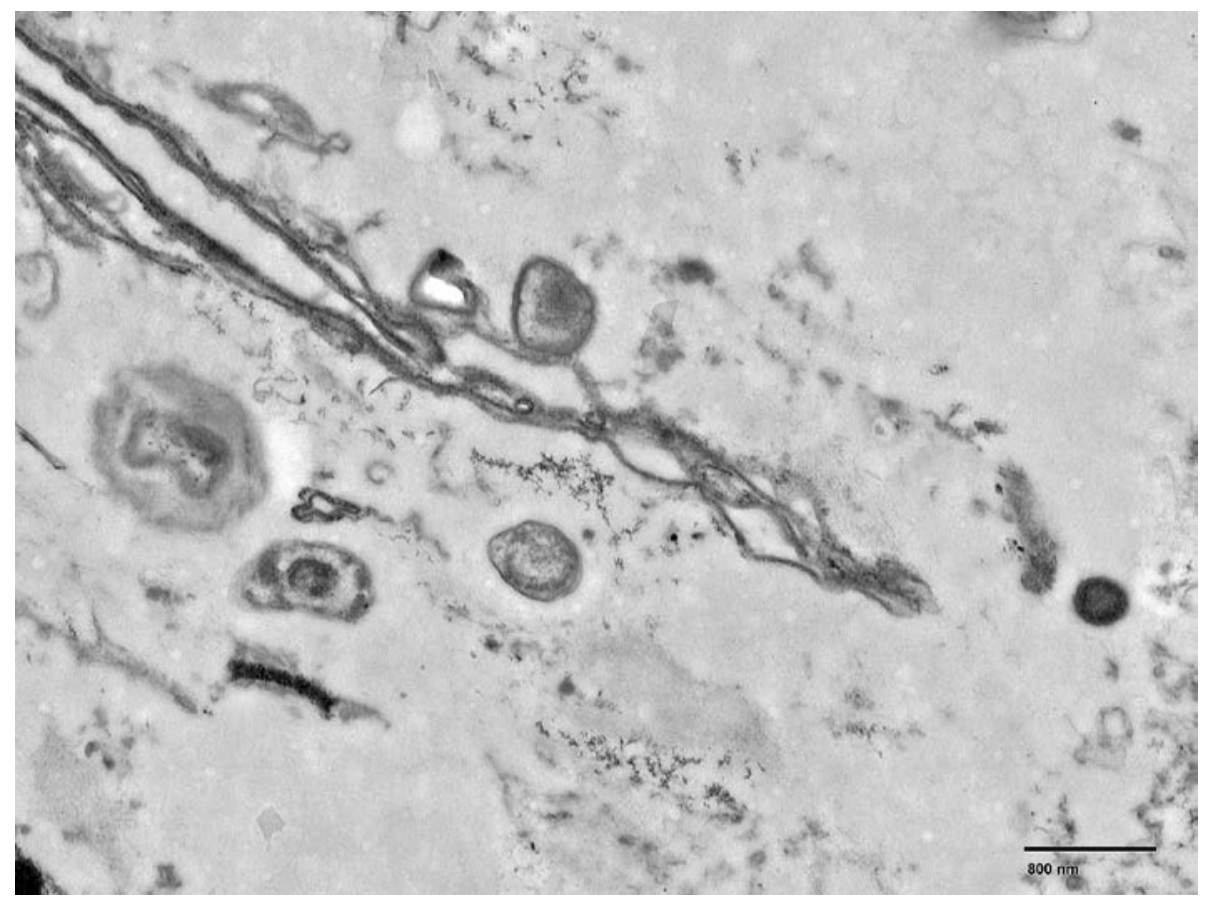



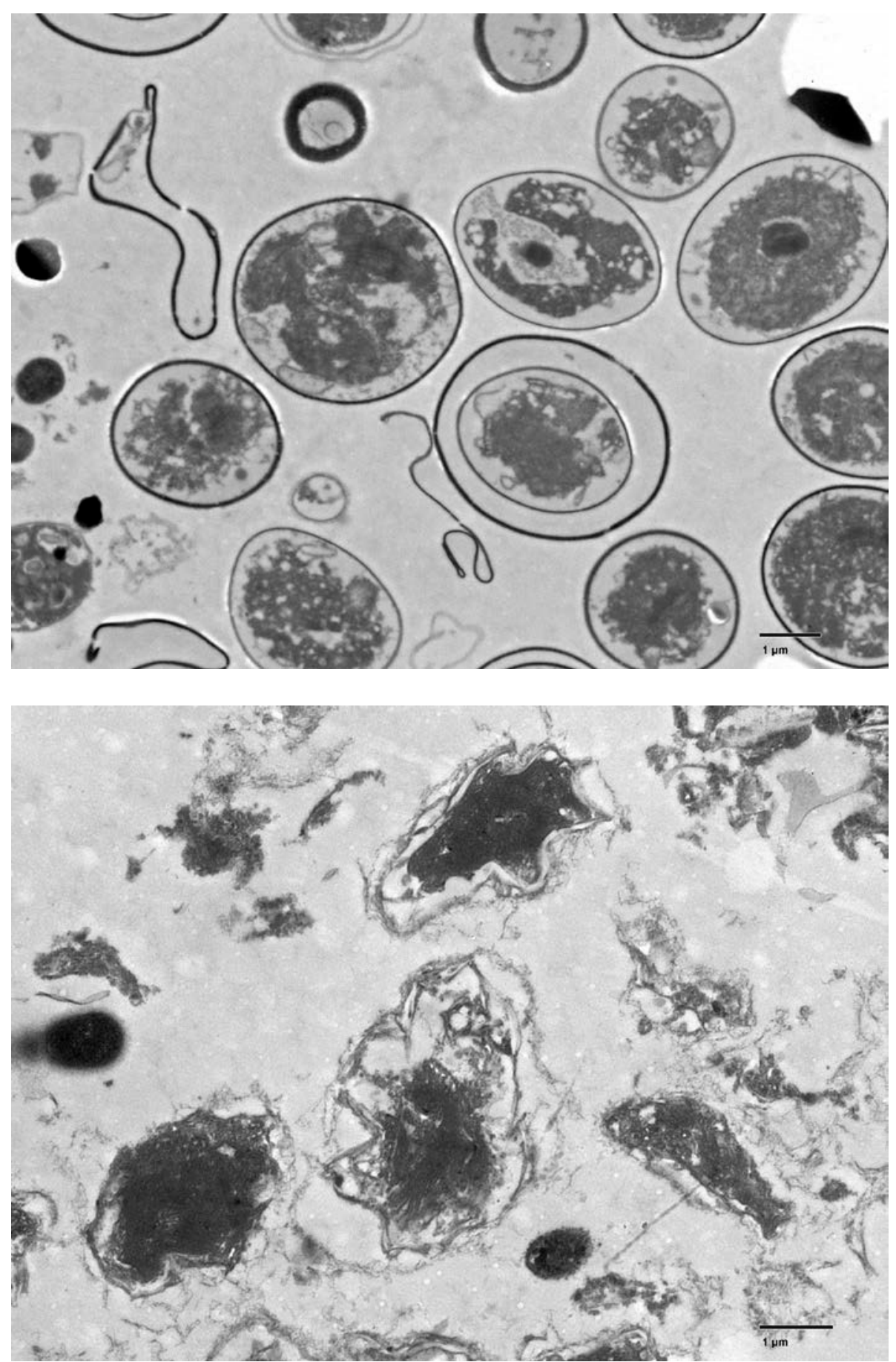

732 Figure S2. TEM images of raw microalgae: (A) Spirulina, (B) Nannochloropsis and (C)

733 Scenedesmus. 

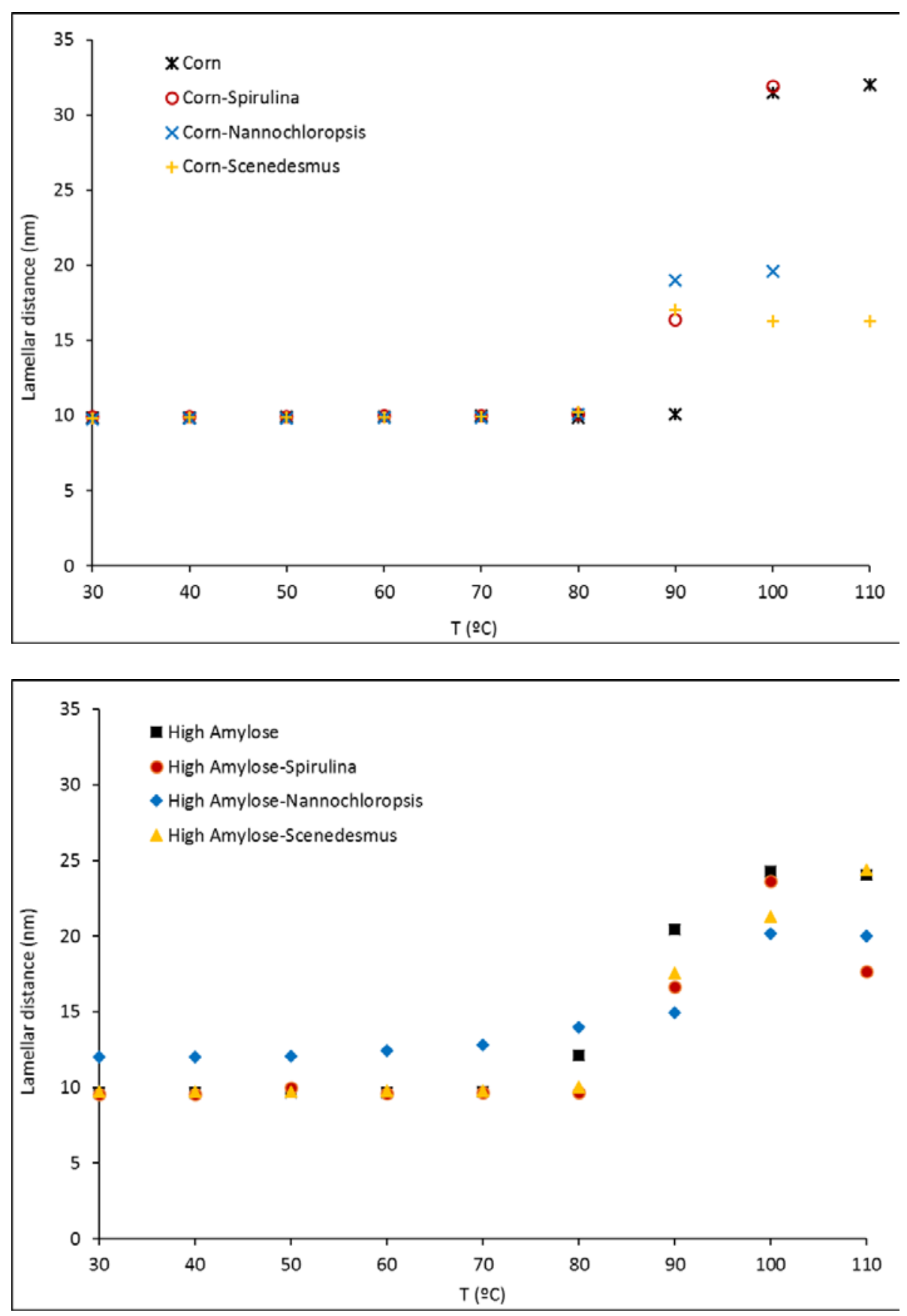

737 Figure S3. Evolution of the lamellar distance extracted from the SAXS fitting parameters

738 for the corn starch (A) and high amylose starch (B) samples. 